\title{
Computability structures, simulations and realizability
}

\author{
John Longley
}

January 10, 2013

\begin{abstract}
We generalize the standard construction of realizability models (specifically, of categories of assemblies) to a wide class of computability structures, broad enough to embrace models of computation such as labelled transition systems and process algebras. We consider a general notion of simulation between such computability structures, and show how these simulations correspond precisely to certain functors between the realizability models. Furthermore, we show that our class of computability structures has good closure properties - in particular, it is 'cartesian closed' in a slightly relaxed sense. Finally, we investigate some important subclasses of computability structures and of simulations between them. We suggest that our 2-category of computability structures and simulations may offer a useful framework for investigating questions of computational power, abstraction and simulability for a wide range of models.
\end{abstract}

\section{Introduction}

The purpose of this paper is to present a rather broad generalization of some ideas and results from the theory of realizability models: in particular, the construction of a category of assemblies over a given model, and a notion of simulation of one model in another. Our theory is general enough to be applicable to a wide range of models of computation from many areas of computer science; our suggestion is that this may provide a useful framework for studying very general questions of computational power, abstraction and simulability.

\section{$1.1 \quad$ Background}

Let us begin with a brief review of the ideas that we wish to generalize. In the standard account of realizability models (as presented e.g. in [23]), one begins with a partial combinatory algebra (or PCA) A - a structure which one may loosely regard as some kind of 'abstract machine' or 'untyped model of computation'. From this, one builds a category such as the category of assemblies $\mathbf{A s m}(A)$, which can be thought of as a 'universe of computable functions' arising from $A$ : its objects can be regarded as 'datatypes' that can be represented or implemented on $A$, and its morphisms can be seen as ' $A$-computable maps' between such datatypes. (We shall review the precise definition of $\operatorname{Asm}(A)$ in $\operatorname{Section} 2$ below.) Categories of this form possess a very rich mathematical structure and have proved interesting from several points of view: for instance, as categorical models corresponding to Kleene-style realizability interpretations of intuitionistic logic [9]; as models for powerful 
polymorphic type systems [17]; or as universes within which certain 'sets' carry an intrinsic domain-like structure [10, 21].

In [12], a notion of morphism between PCAs was introduced which interacts well with the Asm construction. Informally, an applicative morphism $A \longrightarrow B$ is a way of simulating or implementing the abstract machine $A$ on the machine $B$; in many cases it makes sense to think of $A$ here as modelling computation at a 'more abstract' level than $B$. Such a simulation induces a functor $\mathbf{A} \mathbf{s m}(A) \rightarrow \mathbf{A s m}(B)$; moreover, one can give a precise categorical characterization of those functors that arise in this way up to natural isomorphism. In this way, we obtain a tight correspondence between the (2-)category of PCAs and applicative morphisms (which we call $\mathcal{P C A}$ ) and a certain $(2$-) category of categories (again, this material will be reviewed in Section 2). We shall henceforth refer to this result as the correspondence theorem.

Our 2-category $\mathcal{P C A}$ gives rise to a notion of equivalence between PCAs. Informally, two PCAs $A, B$ are equivalent if there are simulations $A \longrightarrow B$ and $B \longrightarrow A$ which are 'mutually inverse up to computable translation'. This notion highlights some interesting differences in 'computational strength' between well-known models of computation: for example, the PCA consisting of the natural numbers with Kleene application turns out to be inequivalent to the PCA of untyped $\lambda$-terms modulo $\beta$-equality, even though these models have the same computational power as measured by the functions $\mathbb{N} \rightarrow \mathbb{N}$ that they can compute. ${ }^{1}$

A consequence of the above theory is that $A$ and $B$ are equivalent if and only if $\mathbf{A s m}(A)$ and $\operatorname{Asm}(B)$ are equivalent as categories. This suggests that we may in some sense view equivalent PCAs as just alternative presentations of the same underlying 'notion of computability', and that the category of assemblies gives us a kind of presentation-invariant embodiment of what it is that they have in common. (See however Section 2.1 for an important caveat on this idea.) Indeed, if one's main interest is in the PCAs themselves and their interrelationships, one can see the correspondence theorem as offering some sort of vindication of the definition of the 2 -category $\mathcal{P C A}$ (and of the resulting notion of PCA equivalence).

All this is a pleasing enough story as far as it goes, and the correspondence theorem we have mentioned is mathematically satisfying. However, this story might be felt to be of limited interest on at least two accounts:

- The vast majority of 'models of computation' studied in computer science today do not naturally take the form of PCAs. However, the general concepts of 'computational power', 'simulation' and 'levels of abstraction' would intuitively seem to be of much more general significance, so it is natural to wonder whether our mathematical framework for discussing these concepts could be broadened to encompass other kinds of models of computation, such as labelled transition systems or process calculi.

- The 2-category $\mathcal{P C} \mathcal{A}$ turns out to be relatively poor in 2-categorical structure. In the interests of coming up with a mathematically fruitful theory, it is natural to ask whether we can do better.

\footnotetext{
${ }^{1}$ This example is explained in detail in [12, Chapter 3]. Briefly, each of these models may be simulated in the other, but the problem is that the simulations cannot be 'essentially mutually inverse' in the required sense, owing to the fact that the $\lambda$-calculus is in some way 'more abstract' than the Kleene model.
} 
As regards the first of these points, some progress was made in [13, 14, 11], where it was shown that the basic theory can be straightforwardly generalized to a setting of typed PCAs, of which ordinary (untyped) PCAs form a special case. This greatly extends the scope of the theory: for instance, the typed PCA framework embraces all the cartesian closed categories used in denotational semantics, as well as a wealth of syntactic models for languages such as PCF and extensions thereof. This version of the theory was presented more fully in [16], where the typed PCA framework played a crucial role in an investigation of higher order computability notions. A further generalization to 'linear' (in contrast to 'intuitionistic') structures was considered by Hoshino in [8].

Even so, the typed PCA framework comes nowhere near to embracing the enormous variety of 'models of computation' currently studied in computer science as a whole. Moreover, from the point of view of categorical structure, the 2-category of typed PCAs is scarcely better than our original 2-category of PCAs. We may therefore still ask whether it is possible to do better on both these scores.

\subsection{Content of the paper}

Our purpose in this paper is to offer a significantly more general framework for the study of models of computation and the 'notions of computability' they embody. Specifically, we shall define a rather wide 2-category $\mathcal{C S} \mathcal{S} \mathcal{R} \mathcal{C} \mathcal{T}$ of computability structures and simulations between them - much broader than the 2-category of typed PCAs - in such a way that:

- a version of the Asm construction still makes sense, and an appropriate generalization of the correspondence theorem goes through;

- a far wider range of models from across computer science are admitted - for example, labelled transition systems and process calculi;

- the class of computability structures has better closure properties than that of (untyped or typed) PCAs.

Our work is motivated by both mathematical and computer science considerations. From a purely mathematical point of view, it can be seen as a deeper investigation into the correspondence theorem first established in the PCA setting: just how far does this phenomenon extend, and what mathematical machinery is needed to make it work? Moreover, the identification of a class of models with richer closure properties is of mathematical interest in its own right, and perhaps an indication that we are ploughing fertile ground. From a computer science point of view, our hope is to offer a general framework for a study of 'computability' and 'simulations' that does justice to the diversity of computation models currently in use. Much of the richness of computer science, after all, comes from the use of a wide variety of modelling styles to study computational systems at many different levels of description or abstraction. A general framework for talking about these modelling styles and their interrelationships therefore holds out the hope of bringing some degree of unification to hitherto disparate parts of the subject.

The key idea behind our generalization is a switch from a 'higher order' to a 'first order' style of modelling. Typed PCAs naturally model higher order flavours of computation, in the sense that a 'computable operation' from a type $\sigma$ to a type $\tau$ is a value of some type 
$(\sigma \rightarrow \tau)$ which may itself serve as input to other computable operations. In the first order style of modelling, data values are rigidly separated from the computable operations that act on them, and only later do we show how the higher order situation may be conveniently recovered as a special case of this. Indeed, one of the main goals of this paper is to show how surprisingly much of the basic machinery of realizability models can be smoothly adapted to this 'flattened' first order setting.

Two major omissions should be noted at the outset. First, our focus in this paper is mostly on setting up the general framework, and there will be relatively little investigation into particular models. Although in Section 3 we shall briefly indicate some of the main sources of examples we have in mind, and present one non-trivial example of a simulation in detail, it would require a far deeper study of specific models from diverse areas of computer science to substantiate our claims regarding the unifying potential of our framework. Secondly, whilst we shall pay some attention to the categorical structure of $\mathbf{A s m}(\mathrm{C})$ for various kinds of model C, we shall barely touch on the kinds of logical constructs that such categories allow us to interpret. Insofar as the interpretation of logic was a key motivation for the study of realizability in the first place, this might appear as a serious deficiency. On the other hand, readers experienced in categorical logic will have little difficulty in seeing broadly what kinds of logical structure our categories support.

The main body of the paper is structured as follows. In Section 2 we give a brief technical summary of the relevant parts of the existing theory of untyped and typed PCAs. In Section 3 we define our main category of interest: the 2-category $\mathcal{C S T} \mathcal{R U C} \mathcal{T}$ of computability structures and simulations. As a non-trivial example, we show how Milner's well-known translation from the lazy $\lambda$-calculus to the $\pi$-calculus $([18])$, can be viewed as a simulation between the relevant computability structures. We also show how any computability structure C gives rise to a 'category of assemblies' $\mathbf{A s m}(\mathrm{C})$, and establish a tight correspondence between simulations $C \longrightarrow D$ and certain functors $\operatorname{Asm}(C) \rightarrow \operatorname{Asm}(D)$, generalizing the existing correspondence theorem for PCAs. In Section 4 we investigate the closure properties of $\mathcal{C S T} \mathcal{R U C T}$, showing in particular that it is 'almost cartesian closed', in a sense that is good enough to ensure that for any structures $C$ and $D$, the structure $D^{C}$ is uniquely determined up to equivalence. In Section 5 we mention some possible variations on our theory and some interesting subcategories of $\mathcal{C S T} \mathcal{R U C \mathcal { T }}$; in particular we show how the theory of typed PCAs fits into this more general framework. We end in Section 6 with some general discussion and suggestions for further work.

\subsection{Related work}

Our work overlaps significantly with recent and essentially independent work by Cockett and Hofstra $[4,5]$. In both their work and ours, the key idea is to generalize the definition of PCA to something with a typed, first order flavour, and to show how a general notion of 'simulation' may be defined in this setting. Another point in common is the idea that models of computation give rise to categories of certain kinds, in such a way that simulation equivalence corresponds precisely to some well-behaved kind of categorical equivalence. In both cases, then, the basic philosophy is that the category serves as a kind of presentationinvariant embodiment of the underlying 'notion of computability'.

Various differences between [5] and our work should also be noted. Firstly, there is a general difference in spirit: whereas Cockett and Hofstra adopt a thoroughgoing 'categorical' 
approach that provides a detailed and precise axiomatization of the mathematical structure that the theory presupposes, we have opted for a relatively simple-minded 'set theoretic' treatment of models of computation as they concretely appear to the working computer scientist. Secondly, at a technical level, there are various differences in the precise level of generality in which the phenomena in question are studied, even after the categorical framework of [5] has been specialized to a familiar set-theoretic setting. These differences will be discussed as we proceed (see Sections 2.1 and 3.1). Nevertheless, there is a very significant area of overlap in which we are talking about essentially the same things — in particular, it is striking that our notion of 'equivalence of models' turns out to coincide exactly (for models within the overlap) with the lax simulation equivalence of [5].

Thirdly, as regards the categories derived from the computation models, the focus of the two approaches is somewhat different. Whereas our aim is to describe concretely a construction that directly generalizes that of the 'classical' category of assemblies, the aim in $[4,5]$ is to give a categorical analysis of that construction in terms of several smaller steps. Indeed, most of the focus in $[4,5]$ is not on the classical category of assemblies itself, but on an intermediate structure known as a Turing category. One of the main results of [5] is a correspondence theorem for Turing categories, closely analogous to ours for categories of assemblies. We shall resume discussion of this in Section 2 below.

Aside from these differences, the following new technical contributions are specific to the present work. First, we identify a precise categorical characterization of those functors between assembly categories that arise from simulations between computability structures; this enables us to state and prove our version of the correspondence theorem. The key notion here is that of a quasi-regular functor - a generalization of the notion of regular functor to a setting where finite products are not assumed. Secondly, our identification of the 'almost cartesian closed' structure in our 2-category of models is novel. Thirdly, there is an explicit emphasis in our work on the generalization to non-deterministic models of computation such as those arising from concurrency theory, and in some respects our definitions are tuned so as to admit a large class of examples from process algebra and related areas (see Example 3.3(iii) and the subsequent discussion).

Also similar in spirit to our work is the general framework for realizability developed by Hofstra in [7]. Here one starts with a broad 2-category of basic combinatorial objects, whose definition resembles that of our computability structures in some respects. On one axis, Hofstra's framework is more restricted than ours: since his main interest is in what one needs in order to build a realizability topos (via a tripos), he restricts attention to untyped structures rather than general typed ones. Along another axis, however, his framework is broader in that it embraces the notion of ordered PCAs, a generalization of ordinary PCAs introduced in [22]. As shown in [7], this extra generality allows one to unify 'computational' examples with geometric or 'localic' ones. We would expect that most if not all of our theory extends readily to the ordered setting. However, we have chosen to eschew this additional generality for the purpose of the present paper, both because we are not aware of any computationally motivated examples that require it, and because the extra generality of the ordered setting is purchased at the cost of a modest technical overhead: our present interest is not so much in maximizing generality as in minimizing the machinery required for our theory to work.

Finally, we mention the work of Abramsky on 'process realizability' [2] as another attempt to broaden the scope of realizability to embrace concurrent and non-deterministic 
flavours of computation as well as the usual functional or applicative kinds. In [2], the ideas are developed in the setting of (a mild extension of) Milner's CCS. In place of a single 'application' operation as in the theory of PCAs, one considers both a 'left' and a 'right' application, dual to each other, which allows the same process term to act as a 'function' in two entirely symmetrical ways. When transposed to our present setting, an effect of the flattening from 'higher order' to 'first order' is that both these kinds of application are subsumed as instances of the more general concept of computable operation. However, the realizability construction Abramsky considers is interestingly different from the standard one, and exploits the duality inherent in his setup to give a model of Classical Linear Logic. We have not yet investigated whether this idea can be fruitfully applied within our present first order framework.

\section{Acknowledgements}

I am very grateful to Robin Cockett and Pieter Hofstra for the invitation to present this material at the PCARC workshop, for illuminating technical discussions, and for the encouragement to write this paper. I am also grateful to the other PCARC participants for their valuable feedback, and to the anonymous referee for several helpful comments and suggestions.

I have benefited from the use of Paul Taylor's LaTeX package for category theory diagrams in the production of this paper.

\section{Untyped and typed PCAs}

We now briefly review of the existing definitions and results for untyped and typed PCAs; for fuller details see [12] or [23]. Later, in Section 5, we shall recover this material as a special case of our more general theory.

If $e, e^{\prime}$ are mathematical expressions, we write $e \downarrow$ to mean "the value of $e$ is defined"; $e \simeq e^{\prime}$ to mean "if either $e$ or $e^{\prime}$ is defined then so is the other and their values are equal"; and $e \succeq e^{\prime}$ to mean "if $e^{\prime}$ is defined then so is $e$ and their values are equal".

\subsection{Untyped PCAs}

The following definition (with minor differences) appeared in [6]; it generalizes the notion of a total combinatory algebra introduced in [20].

Definition 2.1 An (untyped) partial combinatory algebra, or PCA, is a set $A$ equipped with a (left-associative) partial binary operation $\cdot: A \times A \rightarrow A$ and containing elements $k, s$ such that

$$
k \cdot x \cdot y=x \quad s \cdot x \cdot y \downarrow \quad s \cdot x \cdot y \cdot z \succeq x \cdot z \cdot(y \cdot z)
$$

Here and throughout the paper, we adopt that convention that variables in displayed formulae that are not explicitly introduced elsewhere (in this case, $x, y, z$ ) are regarded as implicitly universally quantified at the start of the displayed formula.

Variations on the above definition are possible. For instance, the definition is often given with ' $\simeq$ ' in place of ' $\succeq$ ' — we may call a PCA strict if this stronger condition is satisfied 
- but the theory we shall present works naturally with our slightly weaker definition. ${ }^{2}$ Furthermore, the condition $s \cdot x \cdot y \downarrow$ is from a certain point of view unnecessary (see [23, Theorem 1.2.3]), although it renders the development of our theory somewhat smoother.

The following definition first appeared in [12]:

Definition 2.2 Let $A, B$ be PCAs.

(i) An applicative morphism $\gamma: A \longrightarrow B$ is a total relation $\gamma$ from $A$ to $B$ such that for some $r \in B$ we have

$$
\gamma(a, b) \wedge \gamma\left(a^{\prime}, b^{\prime}\right) \wedge a \cdot a^{\prime} \downarrow \Rightarrow \gamma\left(a \cdot a^{\prime}, r \cdot b \cdot b^{\prime}\right)
$$

(ii) Given $\gamma, \delta: A \longrightarrow B$, we write $\gamma \preceq \delta$, and say there is an applicative transformation from $\gamma$ to $\delta$, if for some $t \in B$ we have

$$
\gamma(a, b) \Rightarrow \delta(a, t \cdot b)
$$

An applicative morphism $A \longrightarrow B$ can be seen as a way of simulating $A$ in $B$; a convenient slogan is "application in $A$ is effective in $B$ ". The condition $\gamma \preceq \delta$ says that $\gamma$-representations for elements of $A$ may be transformed into $\delta$-representations (effectively within $B$ ). It is easy to check that PCAs, applicative morphisms and applicative transformations constitute a preorder-enriched category, which we call $\mathcal{P C} \mathcal{A}$.

We now show how to construct a realizability model over a PCA.

Definition 2.3 For any PCA $A$, the category of assemblies $\mathbf{A} \mathbf{s m}(A)$ is defined as follows.

- Objects are pairs $(X, \Vdash)$ where $X$ is a set and $\Vdash \subseteq A \times X$ is a relation such that for every $x \in X$ there is some $a \Vdash x$.

- Morphisms $(X, \Vdash) \rightarrow\left(Y, \Vdash^{\prime}\right)$ are set-theoretic functions $f: X \rightarrow Y$ such that for some $r \in A$ we have

$$
a \Vdash x \quad \Rightarrow \quad r \cdot a \Vdash f(x)
$$

Identities and composition are as for ordinary functions.

The category $\operatorname{Asm}(A)$ is equipped with an evident forgetful functor $\Gamma_{A}: \operatorname{Asm}(A) \rightarrow \operatorname{Set}$, and also a functor $\nabla_{A}$ : Set $\rightarrow \operatorname{Asm}(A)$ defined as follows: $\nabla_{A}(X)=(X, \Vdash)$ where $a \Vdash x$ for all $a$ and $x$, and $\nabla_{A}(f: X \rightarrow Y)=f$.

It turns out that $\mathbf{A s m}(A)$ is among other things a regular category: that is, it has finite limits and coequalizers of kernel-pairs, and the pullback of a regular epi along any morphism is a regular epi. (An in-depth understanding of this definition is not needed for this paper.) Furthermore, both $\Gamma_{A}$ and $\nabla_{A}$ are regular functors (also known as exact functors): that is to say, they preserve finite limits and coequalizers of kernel-pairs. Note also that $\Gamma_{A}$ is left adjoint to $\nabla_{A}$ and that $\Gamma_{A} \circ \nabla_{A}=$ id.

An applicative morphism $\gamma: A \longrightarrow B$ induces a functor $\mathbf{A s m}(\gamma): \mathbf{A s m}(A) \rightarrow \mathbf{A s m}(B)$ in the following way: $\operatorname{Asm}(\gamma)(X, \Vdash)=\left(X, \Vdash^{\prime}\right)$ where $b \Vdash^{\prime} x$ iff there exists $a \Vdash x$ with $\gamma(a, b)$; and $\mathbf{A} \mathbf{s m}(\gamma)(f)=f$. It is easy to show that $\mathbf{A} \mathbf{s m}(\gamma)$ is a regular functor, and that $\Gamma_{B} \circ \operatorname{Asm}(\gamma) \cong \Gamma_{A}$ and $\operatorname{Asm}(\gamma) \circ \nabla_{A} \cong \nabla_{B}$. Moreover, an applicative transformation $\gamma \preceq \delta$ induces a natural transformation $\operatorname{Asm}(\gamma) \rightarrow \operatorname{Asm}(\delta)$.

Abstracting from this situation, we arrive at the following definition (we write $*$ for horizontal composition):

\footnotetext{
${ }^{2}$ In [23], our 'strict PCAs' are called simply 'PCAs', and our 'PCAs' are referred to as 'lax PCAs'.
} 
Definition 2.4 (i) $A \Gamma \nabla$-regular category is any regular category $\mathcal{C}$ equipped with regular faithful functors $\Gamma: \mathcal{C} \rightarrow$ Set and $\nabla:$ Set $\rightarrow \mathcal{C}$ such that $\Gamma \dashv \nabla$ and $\Gamma \circ \nabla \cong$ id.

(ii) If $\mathcal{C}, \mathcal{D}$ are $\Gamma \nabla$-regular categories, a $\Gamma \nabla$-regular functor $F: \mathcal{C} \rightarrow \mathcal{D}$ is a regular functor such that $\Gamma_{\mathcal{D}} \circ F \cong \Gamma_{\mathcal{C}}$ and $F \circ \nabla_{\mathcal{C}} \cong \nabla_{\mathcal{D}}$. (These isomorphisms are unique when they exist.)

(iii) If $F, G: \mathcal{C} \rightarrow \mathcal{D}$ are $\Gamma \nabla$-regular functors, $a \Gamma \nabla$-natural transformation $\eta: F \rightarrow G$ is a natural transformation such that $\Gamma_{\mathcal{D}} * \eta=\operatorname{id}_{\Gamma_{\mathcal{C}}}$ and $\eta * \nabla_{\mathcal{C}}=\operatorname{id}_{\nabla_{\mathcal{D}}}$. (Both these conditions are automatic in the case $\mathcal{C}=\operatorname{Asm}(A), \mathcal{D}=\operatorname{Asm}(B)$.)

(iv) We write $\Gamma \nabla \mathcal{R E G}$ for the (large) 2-category of $\Gamma \nabla$-regular categories, $\Gamma \nabla$-regular functors and natural transformations between them.

Actually, the $\nabla$ condition in part (ii) is not strictly necessary: a simple cardinality argument (appearing as Lemma 1.6.3 in [23]) shows that for any functor $F: \mathbf{A s m}(A) \rightarrow$ $\operatorname{Asm}(B)$, the condition $\Gamma_{B} \circ F \cong \Gamma_{A}$ automatically implies $F \circ \nabla_{A} \cong \nabla_{B}$.

The tight correspondence between applicative morphisms and regular functors is encapsulated in the following theorem from [12] (see also [23]).

Theorem 2.5 (Correspondence theorem) The Asm construction defines a 2-functor $\mathcal{P C A} \rightarrow \Gamma \nabla \mathcal{R E \mathcal { G }}$ that is locally an equivalence: that is, for any $A, B$ the ordinary functor Asm : $\mathcal{P C} \mathcal{A}[A, B] \rightarrow \Gamma \nabla \mathcal{R E} \mathcal{G}[\operatorname{Asm}(A), \mathbf{A s m}(B)]$ is part of an equivalence of preorders.

Corollary 2.6 $\operatorname{Asm}(A)$ and $\mathbf{A s m}(B)$ are equivalent as categories iff $A, B$ are applicatively equivalent (that is, $A \simeq B$ in the 2-category $\mathcal{P C A}$ ).

The corollary follows because any equivalence of categories $\operatorname{Asm}(A) \simeq \operatorname{Asm}(B)$ may easily be seen to respect the $\Gamma$ and $\nabla$ functors.

Before proceeding to typed PCAs, we pause to discuss a significant choice involved in our definition of applicative morphism: in the terminology of [5], the choice between lax and strict simulations. Our present Definition 2.2(i) corresponds to the lax notion: if $r$ realizes $\gamma: A \longrightarrow B$, we do not require that the degree of partiality of $r$ exactly matches that of application in $A$. To obtain the stricter notion of simulation, we would need to add a condition such as

$$
\gamma\left(a, a^{\prime}\right) \wedge \gamma\left(b, b^{\prime}\right) \wedge r \cdot b \cdot b^{\prime} \downarrow \Rightarrow a \cdot a^{\prime} \downarrow
$$

(The notion of applicative transformation given by Definition 2.2(ii) remains the appropriate one to use in connection with strict simulations - cf. [5, Section 2]). It is known that equivalence of (strict) PCAs via strict applicative morphisms is a stronger condition than equivalence via lax ones (see $\left[4\right.$, Section 5]). ${ }^{3}$

An analogue of our correspondence theorem can also be obtained for the stricter equivalence: whereas lax equivalence coincides with equivalence of the associated categories of assemblies, it is shown in [4] is that strict equivalence coincides with Morita equivalence of Turing categories.

In $[4,5]$, Cockett and Hofstra compellingly argue that strict equivalence is more appropriate where questions of computability are concerned, while the lax notion is better adapted

\footnotetext{
${ }^{3}$ In the context of [4], simulations are also required to be single-valued (cf. Remark 3.6(iii) below). However, this does not affect the present discussion, since both in the lax and strict settings, equivalences may without loss of generality be taken to consist of single-valued simulations (see Corollary 5.18 and the subsequent remark).
} 
to questions of realizability. This is because in realizability it is never a problem if a realizer does 'more' than the job it is designed for, whereas in computability one is often interested in the precise domain of a computable operation. Certainly, if the idea is that equivalent structures should share 'the same' computability theory in some sense, then there will be significant aspects of our structures (such as their theory of m-reducibility) that are stable under strict equivalences but not lax ones. So if we consider models modulo lax equivalence, we are at best dealing with 'computability notions' in a somewhat coarse-grained sense.

Our main reason for concentrating here on the lax notion of simulation is that this appears to us (at present) to be the mathematically more fruitful choice once we generalize to first order structures as in Section 3 below. Indeed, we shall see that the Asm construction and its associated theory generalize well to this broader setting, whereas it seems there is rather little to say by way of a corresponding generalization of Turing categories: most of the interest in these categories (as presented in [4]) seems tied to their higher order nature. We would also add that even our more coarse-grained view would seem to be adequate in practice for mapping out the computability landscape: we do not know of any 'computationally natural' examples of models that are laxly not strictly equivalent.

\subsection{Typed PCAs}

We now briefly sketch the generalization of the above theory to typed PCAs as in [13, 14]. (Note that typed PCAs were called 'partial combinatory type structures' in [14].) The essential difference from untyped PCAs is that in place of a single carrier set $A$, we now allow a whole family of carrier sets indexed by types.

Definition 2.7 (i) A type world is simply an inhabited set $T$ of type names (henceforth types) endowed with right-associative binary operations $*$ and $\Rightarrow$.

(ii) A typed PCA (or TPCA) over a type world $T$ is a family $\left(A_{\sigma} \mid \sigma \in T\right)$ of inhabited sets equipped with partial 'application' operations $\cdot_{\sigma \tau}: A_{\sigma \Rightarrow \tau} \times A_{\sigma} \rightarrow A_{\tau}$ for each $\sigma, \tau \in T$, such that for every $\rho, \sigma, \tau \in T$ there exist elements

$$
\begin{gathered}
k_{\sigma \tau} \in A_{\sigma \Rightarrow \tau \Rightarrow \sigma} \quad s_{\rho \sigma \tau} \in A_{(\rho \Rightarrow \sigma \Rightarrow \tau) \Rightarrow(\rho \Rightarrow \sigma) \Rightarrow \rho \Rightarrow \tau} \\
\text { pair }_{\sigma \tau} \in A_{\sigma \Rightarrow \tau \Rightarrow(\sigma * \tau)} \quad f s t_{\sigma \tau} \in A_{(\sigma * \tau) \Rightarrow \sigma} \quad \text { snd }_{\sigma \tau} \in A_{(\sigma * \tau) \Rightarrow \tau}
\end{gathered}
$$

satisfying the following for all appropriately typed $x, y, z$ :

$$
\begin{aligned}
& k \cdot x \cdot y=x \quad s \cdot x \cdot y \downarrow \quad s \cdot x \cdot y \cdot z \succeq x \cdot z \cdot(y \cdot z) \\
& \text { fst } \cdot(\text { pair } \cdot x \cdot y)=x \quad \text { snd } \cdot(\text { pair } \cdot x \cdot y)=y
\end{aligned}
$$

Note that TPCAs are inherently higher order: an 'operation' that maps values of type $\sigma$ to values of type $\tau$ is itself a value of type $\sigma \Rightarrow \tau$. In the study of higher order computability we are often interested in the type world freely generated from some set of basic type names by means of $*$ and $\Rightarrow$; a TPCA over such a type world may be called a simply typed PCA. Note also that an untyped PCA is just a TPCA over the singleton type world $\mathbf{1}$.

The remaining definitions from Section 2.1 can be straightforwardly adapted to the TPCA setting by means of suitable type decorations. For instance, if $A, B$ are TPCAs over type worlds $T, U$ respectively, an applicative morphism $\gamma: A \longrightarrow B$ consists of a function 
$\gamma: T \rightarrow U$ together with a family of total relations $\gamma_{\sigma}$ from $A_{\sigma}$ to $B_{\gamma \sigma}$, such that each application operation ' $\sigma \tau$ in $A$ is 'tracked' by some $r \in B_{\gamma(\sigma \Rightarrow \tau) \Rightarrow \gamma \sigma \Rightarrow \gamma \tau}$ in the sense of Definition 2.2(i). With the evident definition of an applicative transformation $\gamma \preceq \delta$, this yields a preorder-enriched category $\mathcal{T P C} \mathcal{A}$ of which $\mathcal{P C} \mathcal{A}$ is a full sub-2-category.

An assembly over a TPCA $A$ consists of a triple $(X, \sigma, \Vdash)$ where $X$ is a set, $\sigma$ is a type, and $\Vdash \subseteq A_{\sigma} \times X$ is a relation such that for every $x \in X$ there is some $a \Vdash X$. A morphism $(X, \sigma, \Vdash) \rightarrow\left(Y, \tau, \Vdash^{\prime}\right)$ of such assemblies is just a function $X \rightarrow Y$ that is tracked by some $r \in A_{\sigma \Rightarrow \tau}$ in the obvious way. Once again, the category $\operatorname{Asm}(A)$ of assemblies over a TPCA $A$ turns out to be a $\Gamma \nabla$-regular category, and the Asm construction yields a 2-functor $\mathcal{T} \mathcal{P C} \mathcal{A} \rightarrow \Gamma \nabla \mathcal{R E} \mathcal{G}$ which is locally an equivalence and extends the 2-functor $\mathcal{P C A} \rightarrow \Gamma \nabla \mathcal{R E} \mathcal{G}$ described earlier. As a corollary, the categories $\operatorname{Asm}(A), \mathbf{A s m}(B)$ are equivalent if $A \simeq B$ in the 2-category $\mathcal{T} \mathcal{P C} \mathcal{A}$. (Note that equivalences are possible between TPCAs over different type worlds - indeed, a major theme of [13] was the identification of equivalences between particular untyped PCAs and particular simply typed ones.)

\section{Computability structures and realizability}

We are now ready to introduce our primary object of study: the 2-category of computability structures (henceforth $C$-structures) and simulations between them. We offer this as a relatively broad framework within which a version of the above theory naturally goes through.

\subsection{Computability structures}

In essence, the notion of computability structure can be seen as a 'first order flattening' of the notion of TPCA, in which data values are sharply distinguished from the computable operations that act on them. Furthermore, our computability structures allow computable operations to be multi-valued or 'non-deterministic'.

Definition 3.1 A C-structure C consists of the following:

- a family $|\mathrm{C}|$ of inhabited sets (regarded as 'datatypes'),

- for each $A, B \in|\mathrm{C}|$, a set $\mathrm{C}[A, B]$ of relations from $A$ to $B$ (regarded as 'computable operations', possibly partial and possibly multi-valued)

such that

- for each $A \in|\mathrm{C}|$ there exists $i \in \mathrm{C}[A, A]$ such that $i(a, a)$ for all $a \in A$ (we call such an $i$ a superidentity relation on $A$ );

- if $r \in \mathrm{C}[A, B]$ and $s \in \mathrm{C}[B, C]$, there exists $t \in \mathrm{C}[A, C]$ such that if $r(a, b)$ and $s(b, c)$ then $t(a, c)$ (we call such a relation $t$ a supercomposite of $r$ and $s$ ).

A few technical remarks on this definition are in order. 
Remarks 3.2 (i) It would arguably be more principled to draw a distinction between names of types and their sets of values - that is, to define a C-structure over a set $T$ of type names to be a family of sets $\left(A_{\tau} \mid \tau \in T\right)$ together with certain relations between them, in the spirit of Definition 2.7. This would, for example, allow distinct type names to be assigned the same set of values. However, no interesting extra generality is at stake here, since we may simply relabel elements of datatypes if necessary, and we prefer to avoid the extra notational clutter that such an approach would entail.

(ii) Related to this is our decision to require our datatypes to be inhabited. This is more a matter of stylistic preference than mathematical necessity: all of our main results can be made to go through in the presence of empty types. However, if one empty type were admitted, we would also have to allow C-structures with several unrelated empty types (e.g. to uphold the product construction of Proposition 4.1); and in the absence of type names our framework cannot support these. As it is, we prefer to sidestep these annoying complications by outlawing empty types altogether. Note, however, that the empty C-structure $\mathbf{0}$ (with no datatypes at all) is admitted by Definition 3.1.

(iii) The fact that we only require 'superidentity' and 'supercomposite' relations is a feature shared with [7], though not with [5] where the ambient categorical framework imposes the existence of genuine identities and composites. In the case of superidentities, the extra generality is probably a mere curiosity, since we do not know of any natural examples where proper identities are not present. In the case of composition, however, the additional generality is certainly significant: as we shall shortly see, there are natural examples in which supercomposites are present but ordinary relational composites are not.

The decision to work with these 'super' notions can also be seen as consonant with the decision to use $\succeq$ rather than $\simeq$ in the definition of PCA. Following our terminology for PCAs, C-structures with proper identities and composition may be referred to as strict.

(iv) For readers familiar with TPCAs, it might appear surprising that our 'computable operations' are essentially extensional objects: there would seem to be no provision for distinct 'operations' that induce the same relation on data values. This is because our 'computable operations' generalize the notion of TPCA elements only in their role as the left argument in an application $a \cdot b$ - and for this purpose, only their extension is relevant. We shall see later (Definition 5.22) that when computable operations may themselves be represented by data values, intensional distinctions are indeed allowed for as in TPCAs.

(v) Let us note how the above definition relates to the approach taken in [5]. There, the basic notion of 'model of computation' is that of a category $\mathcal{D}$ equipped with a functor $F: \mathcal{D} \rightarrow \mathcal{C}$ where $\mathcal{C}$ is a restriction category (a 'category of partial maps' in a certain abstract sense). No real generality is lost by assuming $F$ is faithful. The leading example of a restriction category is the category of sets and partial functions, and in this case such models amount to strict C-structures in which all computable operations are single-valued. We shall consider this important class of structures in Section 5.2, under the name of deterministic C-structures. If $\mathcal{C}$ is taken to be the category Rel of sets and relations, we obtain in essence the class of all strict C-structures.

One may also take $\mathcal{C}$ to be the category of sets and total functions (with a trivial restriction structure). Indeed, given any category $\mathcal{C}$ with a terminal object 1 , we may form the C-structure whose datatypes are the sets $\operatorname{Hom}(1, X)$ for $X \in \mathcal{C}$, with computable operations induced by morphisms in $\mathcal{C}$ via composition.

(vi) It is natural to ask whether we can 'categorify' our notion of (lax) C-structure by 
considering a category $\mathcal{D}$ endowed with a lax functor $F: \mathcal{D} \rightarrow$ Rel:

$$
\operatorname{id}_{F(X)} \subseteq F\left(\operatorname{id}_{X}\right) \quad F(g) \circ F(f) \subseteq F(g f)
$$

Indeed, the image in Rel of any such $F$ is in essence a C-structure. However, there are also $\mathrm{C}$-structures that do not arise in this way. For instance, consider the $\mathrm{C}$-structure $\mathrm{C}$ with four datatypes $A=\{a\}, B=\{b\}, C=\{c\}$ and $D=\left\{d, d^{\prime}\right\}$, and with operations as follows (alongside the four identity operations):

$$
\begin{array}{lll}
\{a \mapsto b\} \in \mathrm{C}[A, B] & \emptyset \in \mathrm{C}[B, C] & \{c \mapsto d\} \in \mathrm{C}[C, D] \\
\{a \mapsto c\} \in \mathrm{C}[A, C] & \left\{b \mapsto d^{\prime}\right\} \in \mathrm{C}[B, D] & \{a \mapsto d\},\left\{a \mapsto d^{\prime}\right\} \in \mathrm{C}[A, D]
\end{array}
$$

Suppose $C$ were the image of a lax functor $F: \mathcal{D} \rightarrow$ Rel, where $F(f)=\{a \mapsto b\}, F(g)=\emptyset$ and $F(h)=\{c \mapsto d\}$. Then we must have $F(g f)=\{a \mapsto c\}$ whence $F(h g f)=\{a \mapsto d\}$; on the other hand, $F(h g)=\left\{b \mapsto d^{\prime}\right\}$ whence $F(h g f)=\left\{a \mapsto d^{\prime}\right\}$.

As the above remarks show, the notion of C-structure is mathematically very general indeed. However, our primary interest is in C-structures that model computational processes or operations of some sort. The following selection gives a broad indication of the kinds of examples we have in mind.

Examples 3.3 (i) Any TPCA $A$ over a type world $T$ gives rise to a C-structure A: let $|\mathrm{A}|$ be the family of sets $A_{\sigma}$ where $\sigma \in T$ (relabelling if necessary as per Remark 3.2(i)); and let $\mathrm{A}\left[A_{\sigma}, A_{\tau}\right]$ be the set of partial functions $A_{\sigma} \rightarrow A_{\tau}$ induced by elements of $A_{\sigma \Rightarrow \tau}$.

(ii) Given a labelled transition system with set of states $S$, set of labels $L$, and transition relation $\rightarrow \subseteq S \times L \times S$ (with transitive closure $\rightarrow$ ), we may define a C-structure C as follows. Take $|\mathrm{C}|=\{S\}$. For $w \in L^{*}$ any finite sequence of labels, let $r_{w}$ be the relation $\{(x, y) \mid x \stackrel{w}{\rightarrow} y\}$ on $S$, and let $\mathrm{C}[S, S]$ be the set $\left\{r_{w} \mid w \in L^{*}\right\}$. Clearly this is a C-structure.

(iii) Let $\mathcal{L}$ be some language or calculus comprising the following ingredients:

- A set $T$ of types.

- For each type $\sigma \in T$, an inhabited set $\mathcal{L}_{\sigma}$ of terms of type $\sigma$ (we may think of them as 'closed terms').

- For each $\sigma \in T$, a reflexive, transitive many-step reduction relation $\rightarrow \subseteq \mathcal{L}_{\sigma} \times \mathcal{L}_{\sigma}$.

- A small category $\mathcal{K}$ whose set of objects is $T$, and whose morphisms are regarded as syntactic evaluation contexts, denoted by $K[-]$. This is equipped with a functor $\Theta: \mathcal{K} \rightarrow$ Set such that $\Theta(\sigma)=\mathcal{L}_{\sigma}$ for each $\sigma \in T$; we write $\Theta(K[-])(M)$ as $K[M]$. We also impose the 'evaluation context condition' that $M \rightarrow M^{\prime}$ implies $K[M] \rightarrow K\left[M^{\prime}\right]$ for each $K[-]$ in $\mathcal{K}$.

- For each $\sigma \in T$, an inhabited subset $\mathcal{V}_{\sigma} \subseteq \mathcal{L}_{\sigma}$ of terms designated as values.

Given such a language $\mathcal{L}$, we may define a $\mathrm{C}$-structure $\mathrm{C}_{\mathcal{L}}$ as follows. Let $\left|\mathrm{C}_{\mathcal{L}}\right|$ be the family of all sets $\mathcal{V}_{\sigma}$ for $\sigma \in T$ (again relabelling if necessary as per Remark 3.2(i)). For any $K[-]: \sigma \rightarrow \tau$ in $\mathcal{K}$, let $r_{K} \subseteq \mathcal{V}_{\sigma} \times \mathcal{V}_{\tau}$ be the relation $\{(M, N) \mid K[M] \rightarrow N\}$, and take $\mathrm{C}_{\mathcal{L}}\left[\mathcal{V}_{\sigma}, \mathcal{V}_{\tau}\right]=\left\{r_{K} \mid K[-]: \sigma \rightarrow \tau\right\}$. It is easy to check that $\mathrm{C}_{\mathcal{L}}$ is endowed with identities and supercomposition of relations. 
From example (iii) we may now see the prototypical reason why a naturally arising Cstructure need not be closed under ordinary relational composition. If $K[M] \rightarrow N$ and $L[N] \rightarrow P$ then $L[K[M]] \rightarrow P$, but the term $L[K[M]]$ might also admit reductions to values that do not proceed via any such $L[N]$. For instance, in a process algebra with parallel composition, if $K[-]=Q \mid-$ and $L[-]=R \mid-$, then $L[K[M]]=R|Q| M$ may admit reductions which depend on $R$ interacting directly with $M{ }^{4}$

To give further insight into how the machinery of Example 3.3(iii) works in practice, we briefly outline two concrete specializations, which will also feature in Example 3.8 below.

Examples 3.4 (i) Abramsky's lazy $\lambda$-calculus [1] gives rise to a C-structure as follows. Consider the language with a single type whose terms are just the closed terms of untyped $\lambda$-calculus. Let $\rightarrow_{l}$ be the lazy reduction relation generated by

$$
(\lambda x . M) N \rightarrow_{l} M[x \mapsto N] \quad M \rightarrow_{l} M^{\prime} \Rightarrow M N \rightarrow_{l} M^{\prime} N
$$

and let $\rightarrow_{l}$ be its reflexive-transitive closure. As evaluation contexts, we take all syntactic contexts of the form $-N_{1} \ldots N_{k}$ where $k \geq 0$ and the $N_{i}$ are closed terms; clearly these satisfy the evaluation context condition. (Note that contexts $M$ - will not satisfy this condition unless we choose to add a further reduction rule.) Finally, we may take as values all closed terms of the form $\lambda x$.M. We write $\mathrm{L}$ for the resulting C-structure defined as in Example 3.3(iii).

(ii) Likewise, Milner's $\pi$-calculus gives rise to a C-structure. We assume familiarity here with the presentation in [18]. To facilitate the construction of complex process terms, we shall employ a formulation that allows for meta-terms $\Lambda x . P$ in which a single name $x$ has been abstracted. Specifically, terms and meta-terms are generated by the following grammar, where $x, y$ range over names.

$$
\begin{array}{lrll}
\text { Terms } & P, Q & := & \bar{x} y . P \\
\text { Meta-terms } & U & := & \Lambda x . P
\end{array}
$$

Terms and meta-terms are considered modulo a structural congruence relation $\equiv$, defined as in Definition 3.1 of [18] with the additional clauses:

$$
\Lambda x . P \equiv \Lambda y . P[x \mapsto y] \quad(\Lambda x . P) y \equiv P[x \mapsto y] \quad P \equiv P^{\prime} \Rightarrow \Lambda x \cdot P \equiv \Lambda x \cdot P^{\prime}
$$

Thus, every term $P$ is congruent to a $\Lambda$-free one.

A reduction relation $\rightarrow_{p}$ is now defined on terms and meta-terms modulo $\equiv$ as in Definition 3.2 of [18], with the additional clauses:

$$
P \rightarrow p P^{\prime} \Rightarrow \Lambda x \cdot P \rightarrow \rightarrow_{p} \Lambda x \cdot P^{\prime} \quad U \rightarrow_{p} \quad U^{\prime} \Rightarrow U y \Rightarrow_{p} U^{\prime} y
$$

For $\Lambda$-free terms, this clearly coincides with the reduction relation defined in [18].

We may now consider the language whose terms are closed meta-terms $U$, taking $\rightarrow_{p}$ to be the reflexive-transitive closure of $\rightarrow_{p}$ restricted to such meta-terms. As evaluation contexts, we may take the class of unguarded contexts over meta-terms, namely those generated by composition from syntactic contexts of the form

$$
-\mid Q \quad(y)-\quad-y \quad \Lambda x \cdot-
$$

\footnotetext{
${ }^{4}$ As a further example of some historical interest, we mention Kleene's class of (S1)-(S9) computable partial functionals of higher type, which are not closed under ordinary composition but admit supercomposites in the present sense.
} 
The definition of $\rightarrow p$ ensures that these satisfy the evaluation context condition. Finally, as values, we take those meta-terms that cannot be further reduced via $\rightarrow_{p}$.

We write $\mathrm{P}$ for the resulting $\mathrm{C}$-structure.

\subsection{Simulations}

Next, we introduce the notion of a simulation of one C-structure in another; this generalizes the notion of applicative morphism introduced in Section 2.1.

Definition 3.5 Let C, D be C-structures.

(i) A simulation $\gamma: \mathrm{C} \longrightarrow \mathrm{D}$ consist of:

- a function $A \mapsto \gamma A:|\mathrm{C}| \rightarrow|\mathrm{D}|$,

- for each $A \in|C|$, a total relation $\gamma_{A}$ from $A$ to $\gamma A$

such that every relation $r \in \mathrm{C}[A, B]$ is tracked by some $r^{\prime} \in \mathrm{D}[\gamma A, \gamma B]$ : that is,

$$
r(a, b) \wedge \gamma_{A}\left(a, a^{\prime}\right) \Rightarrow \exists b^{\prime} \cdot r^{\prime}\left(a^{\prime}, b^{\prime}\right) \wedge \gamma_{B}\left(b, b^{\prime}\right)
$$

(ii) If $\gamma, \delta$ are simulations $\mathrm{C} \longrightarrow \mathrm{D}$, we say $\gamma$ is transformable to $\delta$, and write $\gamma \preceq \delta$, if for each $A \in|\mathrm{C}|$ there exists $t \in \mathrm{D}[\gamma A, \delta A]$ such that

$$
\gamma_{A}\left(a, a^{\prime}\right) \Rightarrow \exists a^{\prime \prime} . t\left(a^{\prime}, a^{\prime \prime}\right) \wedge \delta_{A}\left(a, a^{\prime \prime}\right)
$$

Remarks 3.6 (i) In accordance with the discussion of Section 2.1, we have opted for a 'lax' rather than a 'strict' notion of simulation; this is also coherent with our decision to give primacy to lax C-structures rather than strict ones. The strict definition would incorporate the condition

$$
\gamma_{A}\left(a, a^{\prime}\right) \wedge r^{\prime}\left(a^{\prime}, b^{\prime}\right) \Rightarrow \exists b . r(a, b) \wedge \gamma_{B}\left(b, b^{\prime}\right)
$$

(ii) The definition of simulation also presents us with a choice regarding the treatment of non-deterministic (i.e., multi-valued) relations. In the above definition, we have taken the 'liberal' approach of requiring only that every possible behaviour of $r$ on an input $a$ can be mirrored by $r^{\prime}$ on any corresponding element $a^{\prime}$; there may be additional behaviours of $r^{\prime}$ on $a^{\prime}$ with no counterpart in $r$. In Section 5.1, we shall consider a stricter notion of tracking, in which the range of possible behaviours of $r^{\prime}$ on $a^{\prime}$ precisely matches that of $r$ on $a$; this yields the notion of a tight simulation. Note that all single-valued simulations are tight, although not all are strict. Note also that the tightness condition is vacuous if in D every relation is single-valued.

(iii) Our definition of simulation is closely related to what is termed a lax total simulation in [5, Section 2.2]. Indeed, if one takes $\mathcal{C}$ to be the base category of sets and partial functions, then lax total simulations over $\mathcal{C}$ are basically single-valued simulations between the corresponding C-structures, and the refinement relation between such simulations coincides with our $\preceq$. Although from our point of view the restriction to single-valued simulations is rather severe and excludes many of our leading examples even in the untyped PCA setting (see e.g. [12, Chapter 3]), it is at least the case that equivalence via single-valued simulations coincides with equivalence via arbitrary ones: see Section 5.3 below.

If $\mathcal{C}$ is taken to be the category of sets and relations, the connection is less clear-cut: every lax total simulation is a simulation in our sense, but not conversely. 
Proposition 3.7 C-structures and simulations (ordered by $\preceq$ ) form a preorder-enriched category, which we denote by $\mathcal{C S T} \mathcal{R U C T}$.

Proof: The identity simulation on a C-structure $\mathrm{C}$ is as expected; note that every relation in $\mathrm{C}$ tracks itself. Composition of simulations is given by 'typewise' relational composition: given $\gamma: \mathrm{C} \rightarrow \mathrm{D}$ and $\delta: \mathrm{D} \rightarrow \mathrm{E}$, the action of $\delta \circ \gamma$ on $|\mathrm{C}|$ is just the composition of $\delta$ and $\gamma$, and for each $A \in|\mathrm{C}|$, the relation $(\delta \circ \gamma)_{A}$ is simply the (forwards) relational composition $\gamma_{A} ; \delta_{\gamma A}$. To see that $\delta \circ \gamma$ is indeed a simulation, suppose $r \in \mathrm{C}[A, B]$. Take $r^{\prime} \in \mathrm{D}[\gamma A, \gamma B]$ tracking $r$ with respect to $\gamma$, and $r^{\prime \prime} \in \mathrm{E}[\delta \gamma A, \delta \gamma B]$ tracking $r^{\prime}$ with respect to $\delta$; then clearly $r^{\prime \prime}$ tracks $r$ with respect to $\delta \circ \gamma$. The unit and associativity laws for simulations are now immediate from the corresponding facts for relations.

Next we check that for any $C$ and $D$, the simulations $C \longrightarrow D$ are preordered by $\preceq$. For any such simulation $\gamma$, the transformation $\gamma \preceq \gamma$ is witnessed at each $A \in \mathrm{C}$ by any superidentity relation $i \in \mathrm{D}[\gamma A, \gamma A]$. Now suppose $\gamma \preceq \delta \preceq \epsilon$. For any $A \in|\mathrm{C}|$, take $t \in$ $\mathrm{D}[\gamma A, \delta A], u \in \mathrm{D}[\delta A, \epsilon A]$ witnessing $\gamma \preceq \delta, \delta \preceq \epsilon$ respectively at $A$, and let $v \in \mathrm{D}[\gamma A, \epsilon A]$ be any supercomposite of $t$ and $u$. Then $v$ witnesses $\gamma \preceq \epsilon$ at $A$.

Finally, we show that composition of simulations is monotone in both arguments with respect to $\preceq$. Firstly, given $\gamma: \mathrm{C} \longrightarrow \mathrm{D}$ and $\delta \preceq \delta^{\prime}: \mathrm{D} \longrightarrow \mathrm{E}$, if $t$ witnesses $\delta \preceq \delta^{\prime}$ at $\gamma A$ then clearly $t$ also witnesses $\delta \circ \gamma \preceq \delta^{\prime} \circ \gamma$ at $A$. Secondly, given $\gamma \preceq \gamma^{\prime}: \mathrm{C} \longrightarrow \mathrm{D}$ and $\delta: \mathrm{D} \longrightarrow \mathrm{E}$, if $t \in \mathrm{D}\left[\gamma A, \gamma^{\prime} A\right]$ witnesses $\gamma \preceq \gamma^{\prime}$ at $A$ then we may take $t^{\prime} \in \mathrm{E}\left[\delta \gamma A, \delta \gamma^{\prime} A\right]$ tracking $t$ with respect to $\delta$; we claim $t^{\prime}$ witnesses $\delta \circ \gamma \preceq \delta \circ \gamma^{\prime}$ at $A$. For suppose $(\delta \circ \gamma)_{A}\left(a, a^{\prime}\right)$; then there exists $a^{*}$ with $\gamma_{A}\left(a, a^{*}\right)$ and $\delta_{\gamma A}\left(a^{*}, a^{\prime}\right)$, so there exists $a^{* *}$ with $t\left(a^{*}, a^{* *}\right)$ and $\gamma^{\prime}\left(a, a^{* *}\right)$. Hence there exists $a^{\prime \prime}$ with $t^{\prime}\left(a^{\prime}, a^{\prime \prime}\right)$ and $\delta\left(a^{* *}, a^{\prime \prime}\right)$, whence $\left(\delta \circ \gamma^{\prime}\right)_{A}\left(a, a^{\prime \prime}\right)$ as required.

We conclude this subsection with some examples of simulations.

Examples 3.8 (i) Suppose $A, B$ are TPCAs and $\gamma: A \longrightarrow B$ an applicative morphism in the sense of Section 2.2. Let $\mathrm{A}, \mathrm{B}$ be the $\mathrm{C}$-structures arising from $A, B$ as in Example 3.3(i). Then $\gamma$ may clearly be viewed as a simulation $A \longrightarrow B$. Since the preorder $\preceq$ on applicative morphisms clearly agrees with that on simulations, we obtain an inclusion 2-functor $\mathcal{T P C A} \rightarrow \mathcal{C S} \mathcal{T} \mathcal{R U C T}$.

The question of which simulations $A \longrightarrow B$ arise from applicative morphisms in this way will be addressed in Section 5.5.

(ii) Suppose $(S, L, \rightarrow)$ and $\left(S^{\prime}, L, \rightarrow^{\prime}\right)$ are labelled transition systems over the same set $L$ of labels, and let $C, C^{\prime}$ be the corresponding C-structures as in Example 3.3(ii). A simulation $\gamma: C \longrightarrow C^{\prime}$ is then just a certain kind of relation $\gamma \subseteq S \times S^{\prime}$; we say $\gamma$ respects labels if $r_{w^{\prime}}^{\prime}$ tracks $r_{w}$ implies $w^{\prime}=w$. A label-respecting simulation is thus precisely 'half a bisimulation' in the sense of concurrency theory, and $\gamma$ is a bisimulation iff both $\gamma$ and $\gamma^{o p}$ are label-respecting simulations.

(iii) As a more substantial example, we indicate how Milner's well-known translation from the lazy $\lambda$-calculus to the $\pi$-calculus (see [18]) can be viewed as a simulation $L \longrightarrow P$. In effect, Milner defines a compositional translation $\llbracket-\rrbracket$ from untyped $\lambda$-terms to $\pi$-calculus meta-terms:

$$
\begin{aligned}
\llbracket \lambda x \cdot M \rrbracket & =\Lambda u \cdot u(x) \cdot u(v) \cdot \llbracket M \rrbracket v \\
\llbracket x \rrbracket & =\Lambda u \cdot \bar{x} u
\end{aligned}
$$




$$
\begin{array}{rlrl}
\llbracket M N \rrbracket & =\Lambda u \cdot(v)(\llbracket M \rrbracket v \mid(x) \bar{v} x \cdot \bar{v} u \cdot \llbracket x:=N \rrbracket) & (x \notin F V(N)) \\
\llbracket x:=N \rrbracket & =! x(w) \cdot \llbracket M \rrbracket w
\end{array}
$$

Note that if $M$ is a $\lambda$-abstraction then $\llbracket M \rrbracket$ is a value (i.e. it admits no $\rightarrow p^{- \text {-reductions). }}$

The following 'simulation relation' is introduced in [18, Definition 4.4]. If $L$ is a closed untyped $\lambda$-term and $U$ a closed $\pi$-calculus meta-term, we write $\rho(L, U)$ if there exist $\lambda$ terms $M, N_{1}, \ldots, N_{k}$ and distinct variables $x_{1}, \ldots, x_{k}(k \geq 0)$ with $F V(M) \subseteq x_{1}, \ldots, x_{k}$ and $F V\left(N_{i}\right) \subseteq x_{i+1}, \ldots, x_{k}$ such that

$$
\begin{aligned}
L & \equiv M\left[x_{1} \mapsto N_{1}\right] \cdots\left[x_{k} \mapsto N_{k}\right] \\
P & \equiv \Lambda u .\left(x_{1}\right) \cdots\left(x_{k}\right)\left(\llbracket M \rrbracket u\left|\llbracket x_{1}:=N_{1} \rrbracket\right| \cdots \mid \llbracket x_{k}:=N_{k} \rrbracket\right)
\end{aligned}
$$

Restricting $\rho$ to values, we obtain a total relation $\rho$ from elements of $\mathrm{L}$ to those of $\mathrm{P}$ (note that for any closed $\lambda$-abstraction $L$ we have $\rho(L, \llbracket L \rrbracket)$.)

To see that $\rho$ is a simulation $\mathrm{L} \longrightarrow \mathrm{P}$, it will suffice to show that an L-context $-N$ is tracked by the P-context

$$
\Pi_{N}[-]=\Lambda w \cdot(v)(-v \mid(x) \bar{v} x \cdot \bar{v} w \cdot \llbracket x:=N \rrbracket)
$$

where $x$ is fresh; from this it follows readily that a general L-context $-N_{1} \ldots N_{k}$ is tracked by the corresponding composition of P-contexts. First, a simple calculation shows that if $L$ is a value $\lambda z \cdot L^{\prime}$ and $\rho(L, P)$, then for any closed $N$ we have $\Pi_{N}(P) \rightarrow_{p} P^{\prime}$ for some $P^{\prime}$ with $\rho\left(L^{\prime}[z \mapsto N], P^{\prime}\right)$. Secondly, it is shown by Lemma 4.5 of [18] that

- if $L^{\prime}[z \mapsto N] \rightarrow_{l} L_{1}$ and $\rho\left(L^{\prime}[z \mapsto N], P^{\prime}\right)$, then for some $P_{1}$ we have $P^{\prime} \rightarrow_{p} P_{1}$ and $\rho\left(L_{1}, P_{1}\right)$

- if $\rho\left(L_{1}, P_{1}\right)$ and $L_{1}$ is an abstraction, then for some value $P_{1}^{\prime}$ we have $P_{1} \rightarrow_{p} P_{1}^{\prime}$ and $\rho\left(L_{1}, P_{1}^{\prime}\right)$.

Putting all this together gives that if $L N$ reduces to a value $L_{1}$ and $\rho(L, P)$, then $\Pi_{N}(P)$ reduces to a value $P_{1}^{\prime}$ where $\rho\left(L_{1}, P_{1}^{\prime}\right)$ as required.

\subsection{The Asm construction on $\mathcal{C S T} \mathcal{R U C T}$}

The 'category of assemblies' construction can be naturally generalized to C-structures.

Definition 3.9 Given a C-structure $\mathrm{C}$, we define a category $\operatorname{Asm}(\mathrm{C})$ and a functor $\Gamma_{\mathrm{C}}$ : $\operatorname{Asm}(\mathrm{C}) \rightarrow$ Set as follows:

- An object $X$ in $\operatorname{Asm}(\mathrm{C})$ is a triple $\left(|X|, A_{X}, \Vdash_{X}\right)$ where $|X|$ is a set, $A_{X} \in|\mathrm{C}|$, and $\Vdash_{X} \subseteq A_{X} \times|X|$ is a relation such that for every $x \in|X|$ there exists some a $\Vdash_{X} x$.

- A morphism $f: X \rightarrow Y$ in $\mathbf{A s m}(\mathrm{C})$ is a function $f:|X| \rightarrow|Y|$ that is tracked by some $r \in \mathrm{C}\left[A_{X}, A_{Y}\right]$ : that is,

$$
a \Vdash_{X} x \Rightarrow \exists b \cdot r(a, b) \wedge b \Vdash_{Y} f(x)
$$

Identities and composition are defined as in Set. 
- $\Gamma_{\mathrm{C}}$ is the evident forgetful operation $X \mapsto|X|, f \mapsto f$.

It is easy to see that $\operatorname{Asm}(\mathrm{C})$ is indeed a category and $\Gamma_{\mathrm{C}}$ is a faithful functor. We shall always think of $\operatorname{Asm}(C)$ as coming equipped with the functor $\Gamma_{\mathrm{C}}$; in contrast to the situation for PCAs, we cannot in general recover $\Gamma_{\mathrm{C}}$ from $\operatorname{Asm}(\mathrm{C})$ as the functor $\operatorname{Hom}(1,-)$.

Once again, our notion of 'tracking' here involves a choice regarding the treatment of non-determinism. The choice of definition above is consonant with Definition 3.5; when we consider a stricter notion of simulation in Section 5.1, a more restricted notion of assembly morphism will be appropriate.

We now investigate what structure is present in $\left(\operatorname{Asm}(C), \Gamma_{C}\right)$. For this purpose we introduce the following general notions.

Definition 3.10 Let $\mathcal{C}$ be a category, $\Gamma$ a faithful functor $\mathcal{C} \rightarrow$ Set.

(i) $(\mathcal{C}, \Gamma)$ has subobjects if for any object $X$ in $\mathcal{C}$ and any mono $s: S \mapsto \Gamma(X)$ in Set, there exists a morphism $\bar{s}: Y \rightarrow X$ in $\mathcal{C}$ (necessarily mono) such that $\Gamma(\bar{s})=s$, and for any morphism $f: Z \rightarrow X$ such that $\Gamma(f)$ factors through $s$, there is a unique $g: Z \rightarrow Y$ such that $f=\bar{s} \circ g$.

(ii) $(\mathcal{C}, \Gamma)$ has quotients if for any object $X$ in $\mathcal{C}$ and any epi $q: \Gamma(X) \rightarrow Q$ in Set, there exists a morphism $\bar{q}: X \rightarrow V$ in $\mathcal{C}$ (necessarily epi) such that $\Gamma(\bar{q})=q$, and for any morphism $f: X \rightarrow W$ such that $\Gamma(f)$ factors through $q$, there is a unique $g: V \rightarrow W$ such that $f=g \circ \bar{q}$.

(iii) $(\mathcal{C}, \Gamma)$ has copies if for any $X \in \mathcal{C}$ and $S \in$ Set, there is an object $X \propto S$ in $\mathcal{C}$ equipped with morphisms

$$
\pi: X \propto S \rightarrow X \quad \theta: \Gamma(X \propto S) \rightarrow S
$$

such that for any $f: Z \rightarrow X$ and $\phi: \Gamma(Z) \rightarrow S$ there is a unique $g: Z \rightarrow X \propto S$ with $f=\pi \circ g$ and $\phi=\theta \circ \Gamma(g)$.

(iv) We say $(\mathcal{C}, \Gamma)$ is a quasi-regular category over Set if it has subobjects, quotients and copies.

Note that the universal structures given by this definition, when they exist, are unique up to unique isomorphism. In the context of part (i), any morphism $\bar{s}$ with the required properties is called a lifting of $s$ to $\mathcal{C}$; likewise, in part (ii), we say $\bar{q}$ is a lifting of $q$ to $\mathcal{C}$. In part (iii), an object $X \propto S$ with the required properties is called an $S$-fold copy of $X$.

The name 'quasi-regular' reflects the idea that this is as much of the structure of a regular category as survives in this general setting (where we do not have finite products, for instance). The existence of copies does duty for the functor $\nabla: \operatorname{Set} \rightarrow \operatorname{Asm}(A)$ in the PCA case.

Proposition $3.11\left(\operatorname{Asm}(C), \Gamma_{C}\right)$ is a quasi-regular category over $\mathbf{S e t}$.

Proof: To see that $\left(\operatorname{Asm}(\mathrm{C}), \Gamma_{\mathrm{C}}\right)$ has subobjects, suppose $X \in \mathbf{A s m}(\mathrm{C})$ and $s: S \mapsto$ $|X|$. Let $Y$ be the assembly defined by $|Y|=S, A_{Y}=A_{X}$, and $a \Vdash_{Y} y$ iff $a \Vdash_{X} s(y)$; and let $\bar{s}=s$ (this is tracked by any superidentity for $A_{X}$ ). The universal property is then easy. (Actually, it is clear that such a lifting $\bar{s}$ exists even when $s$ is not mono.) 
For quotients, suppose $X \in \mathbf{A s m}(\mathrm{C})$ and $q:|X| \rightarrow Q$. Let $V$ be the assembly defined by $|V|=Q, A_{V}=A_{X}$, and $a \Vdash_{V} v$ iff there exists $x \in|X|$ with $a \Vdash_{X} x$ and $q(x)=v$; and let $\bar{q}=q$ (this is tracked by any superidentity for $A_{X}$ ). The universal property is easy.

For copies, suppose $X \in \mathbf{A s m}(\mathrm{C})$ and $S \in$ Set. Define the assembly $X \propto S$ by

$$
|X \propto S|=|X| \times S \quad A_{X \propto S}=A_{X} \quad a \Vdash_{X \propto S}(x, s) \text { iff } a \Vdash_{X} x .
$$

Let $\pi: X \propto S \rightarrow X$ be the first projection (this is tracked by any superidentity), and let $\theta:|X \propto S| \rightarrow S$ be the second projection. Once again, the universal property is easy.

Next, we see how the Asm construction may be extended to simulations.

Definition 3.12 Given a simulation $\gamma: \mathrm{C} \longrightarrow \mathrm{D}$, define $\gamma_{*}=\mathbf{A} \mathbf{s m}(\gamma): \operatorname{Asm}(\mathrm{C}) \rightarrow$ $\operatorname{Asm}(\mathrm{D})$ as follows:

- On objects $X$, define $\gamma_{*}(X)$ by $\left|\gamma_{*}(X)\right|=|X|, A_{\gamma_{*}(X)}=\gamma A_{X}$, and $b \Vdash_{\gamma_{*}(X)} x$ iff $\exists a \in A_{X} \cdot \gamma_{A_{X}}(a, b) \wedge a \Vdash_{X} x$.

- On morphisms $f: X \rightarrow Y$, define $\gamma_{*}(f)=f$. Note that if $f$ is tracked by $r \in$ $\mathrm{C}\left[A_{X}, A_{Y}\right]$, and $r$ is itself tracked (with respect to $\gamma$ ) by $r^{\prime} \in \mathrm{D}\left[\gamma A_{X}, \gamma A_{Y}\right]$, then $f: \gamma_{*}(X) \rightarrow \gamma_{*}(Y)$ is tracked by $r^{\prime}$.

Proposition 3.13 (i) $\operatorname{Asm}(\gamma)$ is a functor $\operatorname{Asm}(C) \rightarrow \operatorname{Asm}(D)$, and $\Gamma_{\mathrm{D}} \circ \mathbf{A s m}(\gamma)=\Gamma_{\mathrm{C}}$.

(ii) $\operatorname{Asm}(\gamma)$ preserves subobjects, quotients, and copies.

Proof: All parts are trivial; indeed, the structures described in the proof of Proposition 3.11 are preserved on the nose by $\operatorname{Asm}(\gamma)$.

Finally, we show how Asm acts on transformations between simulations.

Definition 3.14 Given simulations $\gamma \preceq \delta: \mathrm{C} \longrightarrow \mathrm{D}$, let $\operatorname{Asm}(\gamma \preceq \delta)$ denote the natural transformation $\xi: \operatorname{Asm}(\gamma) \rightarrow \operatorname{Asm}(\delta)$ defined by $\xi_{X}=\mathrm{id}_{|X|}: \gamma_{*}(X) \rightarrow \delta_{*}(X)$. (Note that if $t \in \mathrm{D}\left[\gamma A_{X}, \delta A_{X}\right]$ witnesses $\gamma \preceq \delta$ at $A_{X}$, then $t$ tracks $\left.\xi_{X}.\right)$

Since $\operatorname{Asm}(\gamma \preceq \delta)$ is simply the identity at the level of sets, it is clear that it is indeed a natural transformation.

We may now put all this together.

Definition 3.15 Let $\Gamma \mathcal{Q R \mathcal { E }}$ be the (large) 2-category defined as follows:

- Objects are quasi-regular categories over Set.

- Morphisms $\left(\mathcal{C}, \Gamma_{\mathcal{C}}\right) \rightarrow\left(\mathcal{D}, \Gamma_{\mathcal{D}}\right)$ are functors $F: \mathcal{C} \rightarrow \mathcal{D}$ equipped with a natural isomorphism $\imath: \Gamma_{\mathcal{C}} \cong \Gamma_{\mathcal{D}} \circ F$ such that

- F preserves subobjects modulo $\imath:$ that is, if $\bar{s}: Y \rightarrow X$ is a subobject lifting of $s: S \longmapsto \Gamma_{\mathcal{C}}(X)$, then $F(\bar{s}): F(Y) \rightarrow F(X)$ is a subobject lifting of $\imath_{X} \circ s \circ \imath_{Y}^{-1}$;

- F preserves quotients modulo $\imath$ (the definition is similar); 
- F preserves copies modulo $\imath$ : that is, if $(X \propto S, \pi, \theta)$ is an $S$-fold copy of $X$ in C, then $\left(F(X \propto S), F(\pi), \theta \circ \imath_{X \propto S}^{-1}\right)$ is an $S$-fold copy of $F(X)$ in D.

We call such a pair $(F, \imath)$ a quasi-regular functor.

- 2-cells $(F, \imath) \rightarrow(G, \jmath)$ are natural transformations $\alpha: F \rightarrow G$.

The following proposition addresses a couple of fine details. Again, we write $*$ for horizontal composition.

Proposition 3.16 (i) For a quasi-regular functor $(F, \imath)$, the isomorphism $\imath$ is uniquely determined by $F$ (so that $\imath$ may be dropped from the data for a quasi-regular functor).

(ii) If $\alpha$ is any natural transformation $F \rightarrow G$ where $(F, \imath)$ and $(G, \jmath)$ are quasi-regular functors $\left(\mathcal{C}, \Gamma_{\mathcal{C}}\right) \rightarrow\left(\mathcal{D}, \Gamma_{\mathcal{D}}\right)$, then automatically $\left(\Gamma_{\mathcal{D}} * \alpha\right) \circ \imath=\jmath$.

Proof: (i) Suppose $X \in \mathrm{C}$ and $x \in \Gamma_{\mathrm{C}}(X)$; we wish to show that $\imath_{X}(x)$ is uniquely determined. Let 1 be a singleton set and regard $x$ as a morphism $1 \longmapsto \Gamma_{\mathrm{C}}(X)$; lift this to a morphism $\bar{x}: Y \rightarrow X$ in $\mathrm{C}$. Since $F$ preserves subobjects modulo $\imath$, we have that $F(\bar{x}): F(Y) \rightarrow F(X)$ is a subobject lifting of $\imath_{X} \circ x \circ \imath_{Y}$. But the latter is a mapping from a singleton set to $\Gamma_{\mathrm{D}}(F(X))$ that picks out $\imath_{X}(x)$. Hence $\imath_{X}(x)$ must be the element picked out by $\Gamma_{\mathrm{D}}(F(\bar{x}))$.

(ii) Suppose $X \in \mathrm{C}$ and $x \in \Gamma_{\mathrm{C}}(X)$; we wish to show that $\Gamma_{D}\left(\alpha_{X}\right)\left(\imath_{X}(x)\right)=\jmath_{X}(x)$. Again, regard $x$ as a morphism $1 \longmapsto \Gamma_{\mathrm{C}}(X)$ and lift this to a morphism $\bar{x}: Y \rightarrow X$ in C. As in the proof of (i) we have that $\Gamma_{\mathrm{D}}(F(\bar{x}))$ picks out $\imath_{X}(x)$ and $\Gamma_{\mathrm{D}}(G(\bar{x}))$ picks out $\jmath_{X}(x)$. Now applying $\Gamma_{D}$ to the naturality square for $\alpha$ at $\bar{x}$, we have that $\Gamma_{D}\left(\alpha_{X}\right) \circ \Gamma_{\mathrm{D}}(F(\bar{x}))=$ $\Gamma_{\mathrm{D}}(G(\bar{x})) \circ \Gamma_{\mathrm{D}}\left(\alpha_{Y}\right)$; and since $\Gamma_{\mathrm{D}}\left(\alpha_{Y}\right)$ is just a function between singleton sets, this says that $\Gamma_{D}\left(\alpha_{X}\right)\left(\imath_{X}(x)\right)=\jmath_{X}(x)$ as required.

Theorem 3.17 The constructions of Definitions 3.9, 3.12 and 3.14 constitute a 2-functor

$$
\text { Asm }: \mathcal{C S T} \mathcal{R U C T} \rightarrow \Gamma \mathcal{Q R \mathcal { G }}
$$

Proof: In addition to the properties already established, it is trivial that Asm respects identity simulations, composition of simulations, identity transformations and vertical and horizontal composition of transformations.

Clearly, the Asm construction on C-structures agrees up to isomorphism with that on TPCAs (as in Section 2.2) modulo the inclusion 2-functor $\mathcal{T P C A} \rightarrow \mathcal{C S T} \mathcal{R} \mathcal{M C} \mathcal{T}$ noted under Example 3.8(i).

We conclude this subsection with a brief aside on how our notions of subobject and quotient relate to regular monos and epis in $\mathbf{A s m}(\mathrm{C})$ (we leave the verifications as exercises). On the one hand, all equalizers exist in $\operatorname{Asm}(C)$, and our subobjects are precisely the regular monos. On the other hand, all coequalizers exist in $\operatorname{Asm}(C)$, and every regular epi is a quotient morphism, though the converse does not seem to be true in general. However, if $\mathrm{C}$ is endowed with cartesian products (as in Section 5.4), then the notions of quotient and regular epi coincide.

Furthermore, for any simulation $\gamma$, the functor $\operatorname{Asm}(\gamma)$ preserves all equalizers and coequalizers. In the light of Theorem 3.20 below, this means any quasi-regular functor $\operatorname{Asm}(\mathrm{C}) \rightarrow \operatorname{Asm}(\mathrm{D})$ preserves equalizers and coequalizers. 


\subsection{The correspondence theorem}

We now work towards showing that the 2-functor Asm is locally an equivalence: that is, for

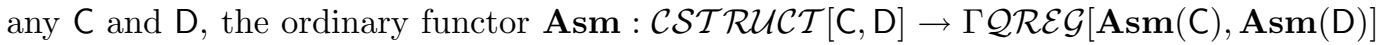
is part of an equivalence of preorders. The following proposition shows that essentially every quasi-regular functor $\mathbf{A s m}(C) \rightarrow \mathbf{A s m}(D)$ arises from some simulation $C \longrightarrow D$.

Proposition 3.18 Let $F:\left(\operatorname{Asm}(\mathrm{C}), \Gamma_{\mathrm{C}}\right) \rightarrow\left(\operatorname{Asm}(\mathrm{D}), \Gamma_{\mathrm{D}}\right)$ be a morphism in $\Gamma \mathcal{Q R \mathcal { G }}$. Then there is a simulation $\gamma: \mathrm{C} \longrightarrow \mathrm{D}$ such that there is a unique natural isomorphism $\operatorname{Asm}(\gamma) \cong F$.

Proof: Given $F$, we define $\gamma$ as follows. For each $A \in|\mathrm{C}|$, let $Z_{A}$ be the 'object of realizers' in $\operatorname{Asm}(\mathrm{C})$ given by $\left|Z_{A}\right|=A, A_{Z_{A}}=A, \Vdash_{Z_{A}}=\operatorname{id}_{A}$. Let $W_{A}=F\left(Z_{A}\right)$, and define $\gamma A=A_{W_{A}}$ and $\gamma_{A}(a, b)$ iff $b \Vdash_{W_{A}} a$.

To see that $\gamma$ is a simulation, suppose $r \in \mathrm{C}[A, B]$; we wish to show $r$ is tracked by some $r^{\prime} \in \mathrm{D}[\gamma A, \gamma B]$. Let $R$ be the relation $r$ viewed as a subset of $\left|Z_{A} \propto B\right|=A \times B$, and lift this to a subobject $Z_{R} \longmapsto Z_{A} \propto B$. Likewise, let $S$ be the opposite of $r$ viewed as a subset of $\left|Z_{B} \propto A\right|=B \times A$, and lift this to a subobject $Z_{S} \longmapsto Z_{B} \propto A$. By the universal property of the latter, the canonical bijection $t: R \rightarrow S$ (the twist map) lifts to a morphism $\tilde{t}: Z_{R} \rightarrow Z_{S}$ which is tracked by $r$ itself:

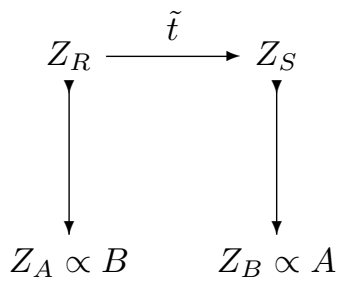

We now apply $F$ to this whole diagram, then replace the relevant copies and subobjects by the isomorphic 'canonical' ones in $\mathbf{A s m}(\mathrm{D})$ (i.e., those described in the proof of Proposition 3.11):

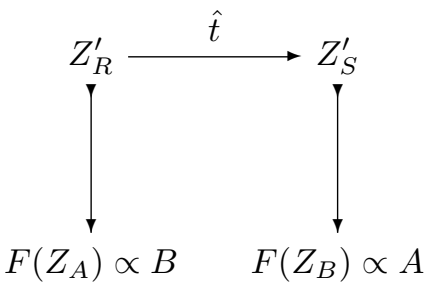

Note that the type of realizers for $Z_{R}^{\prime}$ is that of $F\left(Z_{A}\right)$, that is $\gamma A$, and likewise the type of realizers for $Z_{S}^{\prime}$ is $\gamma B$. So let $r^{\prime} \in \mathrm{D}[\gamma A, \gamma B]$ be any relation tracking $\hat{t}$. We claim that $r^{\prime}$ tracks $r$ with respect to $\gamma$. Indeed, suppose $r(a, b)$ and $\gamma_{A}\left(a, a^{\prime}\right)$. Then $a^{\prime} \Vdash_{Z_{R}^{\prime}}(a, b)$, since $\gamma_{A}$ is by definition extracted from $F\left(Z_{A}\right)$. So there exists $b^{\prime} \Vdash_{Z_{S}^{\prime}}(b, a)=\hat{t}(a, b)$ such that $r^{\prime}\left(a^{\prime}, b^{\prime}\right)$. But now $\gamma_{B}\left(b, b^{\prime}\right)$ by definition of $\Vdash_{Z_{S}^{\prime}}$ and $\gamma_{B}$ (both are induced by $F\left(Z_{B}\right)$ ) and we are done.

It remains to show that $\gamma_{*}=\operatorname{Asm}(\gamma) \cong F$. Consider an arbitrary $X \in \operatorname{Asm}(\mathrm{C})$. We may express $X$ as a quotient of a subobject of $Z_{A} \propto|X|$, where $A=A_{X}$. Specifically, let 
$R$ be the subset of $\left|Z_{A} \propto\right| X||$ corresponding to $\Vdash_{X}$, and let $q: R \rightarrow|X|$ be the surjection given by second projection. These lift to subobject and quotient maps in $\operatorname{Asm}(C)$, giving the diagram

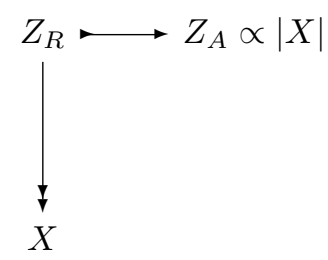

Applying $F$ to this diagram and replacing copies, subobjects and quotients in turn by isomorphic canonical ones, we obtain a diagram

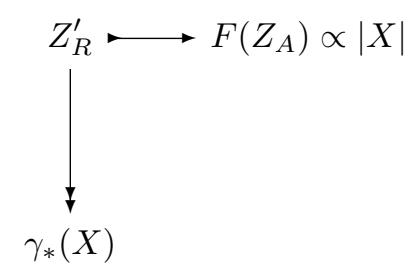

So we obtain an isomorphism $\gamma_{*}(X) \cong F(X)$ which agrees on underlying sets with the given isomorphism $\Gamma_{\mathrm{C}} \cong \Gamma_{\mathrm{D}} \circ F$.

To see that this isomorphism is natural in $X$, it now suffices to note that if $f: X \rightarrow Y$ in $\operatorname{Asm}(\mathrm{C})$ then $\Gamma_{\mathrm{D}}\left(\gamma_{*}(f)\right)=f$, and $\Gamma_{\mathrm{D}}(F(f)) \cong \Gamma_{\mathrm{C}}(f)=f$ via the given isomorphism $\Gamma_{\mathrm{D}} \circ F \cong \Gamma_{\mathrm{C}}$.

We now see how the correspondence works at the level of 2-cells:

Proposition 3.19 Suppose $\gamma, \delta: \mathrm{C} \longrightarrow \mathrm{D}$ are simulations, and suppose $\eta: \gamma_{*} \rightarrow \delta_{*}$ is a natural transformation. Then $\gamma \preceq \delta$ and $\eta=\operatorname{Asm}(\gamma \preceq \delta)$.

Proof: First note that by Proposition 3.16(ii), it is automatic that $\Gamma_{\mathrm{D}} * \eta=\operatorname{id}_{\Gamma_{\mathrm{C}}}$.

For each $A \in|C|$, let $Z_{A}$ be the object of realizers as in the previous proof, and consider $\eta_{Z_{A}}: \gamma_{*}\left(Z_{A}\right) \rightarrow \delta_{*}\left(Z_{A}\right)$. Suppose this is tracked by $t \in \mathrm{D}[\gamma A, \delta A]$. Since $\eta_{Z_{A}}$ is just the identity on $A$, we have

$$
a^{\prime} \Vdash_{\gamma_{*}\left(Z_{A}\right)} a \Rightarrow \exists a^{\prime \prime} . t\left(a^{\prime}, a^{\prime \prime}\right) \wedge a^{\prime \prime} \Vdash_{\delta_{*}\left(Z_{A}\right)} a
$$

That is, $\gamma\left(a, a^{\prime}\right)$ implies $\exists a^{\prime \prime} . t\left(a^{\prime}, a^{\prime \prime}\right) \wedge \delta\left(a, a^{\prime \prime}\right)$. In other words, $t$ witnesses $\gamma \preceq \delta$ at $A$.

Again by Proposition 3.16(ii), it is automatic that $\Gamma_{\mathrm{D}} * \mathbf{A s m}(\gamma \preceq \delta)=\mathrm{id}_{\Gamma_{\mathrm{C}}}$. So $\Gamma_{\mathrm{D}} * \eta=$ $\Gamma_{\mathrm{D}} * \operatorname{Asm}(\gamma \preceq \delta)$, and because $\Gamma_{\mathrm{D}}$ is faithful, this means $\eta=\operatorname{Asm}(\gamma \preceq \delta)$.

We now have everything that is needed for our generalized correspondence theorem, which to some extent validates our definition of $\mathcal{C S T} \mathcal{R U C T}$ :

Theorem 3.20 The 2-functor $\mathbf{A s m}: \mathcal{C S T} \mathcal{R U C T} \rightarrow \Gamma \mathcal{Q R E G}$ is locally an equivalence. 
Corollary 3.21 The C-structures $\mathrm{C}$ and $\mathrm{D}$ are equivalent in $\mathcal{C S T} \mathcal{R U C \mathcal { T }}$ iff there is an equivalence of categories $(F, G): \mathbf{A} \mathbf{s m}(\mathrm{C}) \simeq \operatorname{Asm}(\mathrm{D})$ with $\Gamma_{\mathrm{D}} \circ F \cong \Gamma_{\mathrm{C}}$ and $\Gamma_{\mathrm{C}} \circ G \cong \Gamma_{\mathrm{D}}$.

PROOF: The forwards implication is immediate from Theorem 3.17 (indeed, this yields an equivalence that commutes with $\Gamma$ on the nose). For the reverse, it is easy to see that if $F$ and $G$ are as above then $F$ and $G$ are quasi-regular functors, and so constitute an equivalence in $\Gamma \mathcal{Q R \mathcal { E }}$. It now follows from Theorem 3.20 that $\mathrm{C} \simeq \mathrm{D}$ in $\mathcal{C} \mathcal{S} \mathcal{R} \mathcal{U C} \mathcal{C}$.

\section{Categorical structure in $\mathcal{C S T} \mathcal{R U C T}$}

We now investigate some of the categorical structure available in the 2-category $\mathcal{C S T} \mathcal{R U C T}$. Although our investigation here is far from exhaustive, the picture that emerges is that

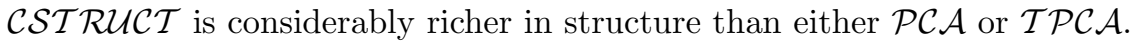

If $X$ and $Y$ are sets of sets, we write $X \otimes Y$ for the set $\{A \times B \mid A \in X, B \in Y\}$.

Proposition $4.1 \mathcal{C S T} \mathcal{R U C T}$ has finite products, enriched with respect to $\preceq$.

Proof: The terminal object $\mathbf{1}$ is the unique C-structure with $|\mathbf{1}|=\{\{*\}\}$. If C, D are C-structures, define $\mathrm{C} \times \mathrm{D}$ by

$$
\begin{aligned}
|\mathrm{C} \times \mathrm{D}| & =|\mathrm{C}| \otimes|\mathrm{D}| \\
(\mathrm{C} \times \mathrm{D})[A \times B, C \times D] & =\mathrm{C}[A, C] \otimes \mathrm{D}[B, D]
\end{aligned}
$$

Clearly $\mathrm{C} \times \mathrm{D}$ is a $\mathrm{C}$-structure, and there are evident projection simulations $\pi_{\mathrm{C}}: \mathrm{C} \times \mathrm{D} \longrightarrow \mathrm{C}$ and $\pi_{\mathrm{D}}: \mathrm{C} \times \mathrm{D} \longrightarrow \mathrm{C}$. Given simulations $\gamma: \mathrm{E} \longrightarrow \mathrm{C}$ and $\delta: \mathrm{E} \longrightarrow \mathrm{D}$, we have a simulation $\langle\gamma, \delta\rangle: \mathrm{E} \longrightarrow \mathrm{C} \times \mathrm{D}$ given by

$$
\begin{aligned}
\langle\gamma, \delta\rangle A & =\gamma A \times \delta A \\
\langle\gamma, \delta\rangle_{A}(e,(c, d)) & \Leftrightarrow \gamma_{A}(e, c) \wedge \delta_{A}(e, d)
\end{aligned}
$$

Clearly this is the unique simulation such that $\pi_{\mathrm{C}} \circ\langle\gamma, \delta\rangle=\gamma$ and $\pi_{\mathrm{D}} \circ\langle\gamma, \delta\rangle=\delta$, and $\langle\gamma, \delta\rangle \preceq\left\langle\gamma^{\prime}, \delta^{\prime}\right\rangle$ iff $\gamma \preceq \gamma^{\prime}$ and $\delta \preceq \delta^{\prime}$.

Proposition $4.2 \mathcal{C S T} R \mathcal{U C T}$ has finite sums, enriched with respect to $\preceq$.

ProOf: The initial object $\mathbf{0}$ is the empty C-structure. As a temporary notation, for any set $A$ we write $A_{i}$ for $\{i\} \times A$. If C, D are C-structures, define $\mathrm{C}+\mathrm{D}$ by

$$
\begin{aligned}
|\mathrm{C}+\mathrm{D}| & =\left\{A_{0}|A \in| \mathrm{C} \mid\right\} \cup\left\{B_{1}|B \in| \mathrm{D} \mid\right\} \\
(\mathrm{C}+\mathrm{D})\left[A_{0}, B_{0}\right] & \cong \mathrm{C}[A, B] \\
(\mathrm{C}+\mathrm{D})\left[A_{1}, B_{1}\right] & \cong \mathrm{D}[A, B] \\
(\mathrm{C}+\mathrm{D})\left[A_{i}, B_{j}\right] & =\emptyset \text { if } i \neq j
\end{aligned}
$$

Clearly $\mathrm{C}+\mathrm{D}$ is a C-structure, and there are evident inclusion simulations $\iota_{\mathrm{C}}: \mathrm{C} \longrightarrow \mathrm{C}+\mathrm{D}$ and $\iota_{\mathrm{D}}: \mathrm{D} \longrightarrow \mathrm{C}+\mathrm{D}$. Given simulations $\gamma: \mathrm{C} \longrightarrow \mathrm{E}$ and $\delta: \mathrm{D} \longrightarrow \mathrm{E}$, there is evidently a unique simulation $[\gamma, \delta]: \mathrm{C}+\mathrm{D} \longrightarrow \mathrm{E}$ such that $[\gamma, \delta] \circ \iota_{\mathrm{C}}=\gamma$ and $[\gamma, \delta] \circ \iota_{\mathrm{D}}=\delta$, and clearly $[\gamma, \delta] \preceq\left[\gamma^{\prime}, \delta^{\prime}\right]$ iff $\gamma \preceq \gamma^{\prime}$ and $\delta \preceq \delta^{\prime}$. 
Although both these results are trivial, Proposition 4.2 already gives us something not available in $\mathcal{P C} \mathcal{A}$ or $\mathcal{T} \mathcal{P C} \mathcal{A}$. If $A, B$ were TPCAs over some $T$, for instance, the evident disjoint union $A+B$ would not (canonically) be a TPCA, since it would lack a type of operations mapping $A_{\sigma}$ to $B_{\tau}$ (where $\sigma, \tau \in T$ ). Most probably, $\mathcal{P C} \mathcal{A}$ and $\mathcal{T P C} \mathcal{A}$ do not have categorical binary sums at all.

More interestingly, it turns out that $\mathcal{C S} \mathcal{T} \mathcal{R U C} \mathcal{T}$ is very nearly cartesian closed: indeed, for any C-structures C, D, an 'exponential' C-structure $\mathrm{D}^{\mathrm{C}}$ exists in a slightly weak sense which is nonetheless sufficient to characterize $D^{C}$ uniquely up to equivalence in $\mathcal{C S T} \mathcal{R U C T}$. Let us begin by making the characteristic property of $\mathrm{D}^{\mathrm{C}}$ precise.

Definition 4.3 $A$ simulation $\gamma: \mathrm{C} \longrightarrow \mathrm{D}$ is single-valued if for any $A \in|\mathrm{C}|$ and $a \in A$, there is a unique $a^{\prime} \in \gamma A$ with $\gamma_{A}\left(a, a^{\prime}\right)$.

Definition 4.4 Suppose C, D are C-structures, and we are given a $C$-structure $\mathrm{F}$ along with a simulation eval $: \mathrm{F} \times \mathrm{C} \longrightarrow \mathrm{D}$. We call (F, eval) a near-exponential for (C, D) if for any $C$ structure $\mathrm{E}$ and simulation $\alpha: \mathrm{E} \times \mathrm{C} \longrightarrow \mathrm{D}$, there is a single-valued simulation $\bar{\alpha}: \mathrm{E} \longrightarrow \mathrm{F}$ such that eval $\circ\left(\bar{\alpha} \times \mathrm{id}_{\mathrm{C}}\right)=\alpha$, and moreover $\bar{\alpha}$ is, up to $\preceq \succeq$, the unique single-valued simulation with this property.

Because single-valued simulations compose and identity simulations are single-valued, it is easy to see that a near-exponential for $(C, D)$ is uniquely determined up to equivalence in $\mathcal{C S T R U C T}$

The rest of this section will be devoted to the proof of the following:

Theorem 4.5 $\mathcal{C S T} \mathcal{R U C T}$ possesses a near-exponential ( $\mathrm{D}^{\mathrm{C}}$, eval) for every $(\mathrm{C}, \mathrm{D})$.

The conceptual significance of this result is far from clear to us: if C and D represent 'models of computation', what on earth does it mean to consider $\mathrm{D}^{\mathrm{C}}$ as a 'model of computation'? At present, the existence of near-exponentials stands as something of a curiosity, albeit a rather striking one which offers evidence for the mathematical richness of our framework.

From now on let C and D be fixed C-structures. We start with the definition of the C-structure $\mathrm{D}^{\mathrm{C}}$.

Definition 4.6 (i) Given any function $U:|\mathrm{C}| \rightarrow|\mathrm{D}|$, we write $\left[\mathrm{C} \longrightarrow{ }_{U} \mathrm{D}\right]$ for the set of simulations $\gamma: \mathrm{C} \longrightarrow \mathrm{D}$ such that $\gamma A=U(A)$ for all $A \in|\mathrm{C}|$.

(ii) A family $F \subseteq\left[\mathrm{C} \longrightarrow{ }_{U} \mathrm{D}\right]$ of simulations is uniformly tracked if for all $A, B \in|\mathrm{C}|$ and $r \in \mathrm{C}[A, B]$, there exists $r^{\prime} \in \mathrm{D}[U(A), U(B)]$ such that $r^{\prime}$ tracks $r$ with respect to every $\gamma \in F:$ that is,

$$
\forall \gamma \in F . \quad r(a, b) \wedge \gamma_{A}\left(a, a^{\prime}\right) \Rightarrow \exists b^{\prime} . r^{\prime}\left(a^{\prime}, b^{\prime}\right) \wedge \gamma_{B}\left(b, b^{\prime}\right)
$$

(Note that if $F$ is uniformly tracked and inhabited then we can recover $U$ from $F$; we write it as $U_{F}$ and call it the underlying type operation of $F$.)

(iii) If $F \subseteq\left[\mathrm{C} \longrightarrow{ }_{U} \mathrm{D}\right]$ and $G \subseteq\left[\mathrm{C} \triangleright_{V} \mathrm{D}\right]$ are each uniformly tracked and inhabited, we say $R \subseteq F \times G$ is a uniform transformation if for all $A \in|C|$ there exists $t \in \mathrm{D}[U(A), V(A)]$ such that for every $(\gamma, \delta) \in R$ we have that $t$ witnesses $\gamma \preceq \delta$ at $A$ : that is,

$$
\forall(\gamma, \delta) \in R . \quad \gamma_{A}(a, b) \Rightarrow \exists c . t(b, c) \wedge \delta_{A}(a, c)
$$

(iv) We now define a $C$-structure $\mathrm{D}^{\mathrm{C}}$ as follows: 
- $\left|\mathrm{D}^{\mathrm{C}}\right|$ is the set of all uniformly tracked and inhabited families $F$ of simulations $\mathrm{C} \longrightarrow \mathrm{D}$.

- For any $F, G \in\left|\mathrm{D}^{\mathrm{C}}\right|, \mathrm{D}^{\mathrm{C}}[F, G]$ is the set of uniform transformations $R \subseteq F \times G$.

Proposition $4.7 \mathrm{D}^{\mathrm{C}}$ is indeed a C-structure.

Proof: To see that $\mathrm{D}^{\mathrm{C}}$ has (super)identities, given any $F \in\left|\mathrm{D}^{\mathrm{C}}\right|$, take $I \subseteq F \times F$ to be the identity relation. Clearly $I$ is a uniform transformation: for any $A \in|C|$, let $i$ be any superidentity on $U_{F}(A)$; then for any $\gamma \in F, i$ witnesses $\gamma \preceq \gamma$ at $A$. So $I \in \mathrm{D}^{\mathrm{C}}[F, F]$.

To see that $\mathrm{D}^{\mathrm{C}}$ has (super)composites, suppose $R \in \mathrm{D}^{\mathrm{C}}[F, G]$ and $S \in \mathrm{D}^{\mathrm{C}}[G, H]$ and let $T$ be the relational composition $R ; S$. We claim $T$ is a uniform transformation. Given $A \in|\mathrm{C}|$, take $t \in \mathrm{D}\left[U_{F}(A), U_{G}(A)\right]$ uniformly witnessing $\gamma \preceq \delta$ at $A$ for all $(\gamma, \delta) \in R$, and take $u \in \mathrm{D}\left[U_{G}(A), U_{H}(A)\right]$ uniformly witnessing $\delta \preceq \epsilon$ at $A$ for all $(\delta, \epsilon) \in S$. Let $v \in \mathrm{D}\left[U_{F}(A), U_{H}(A)\right]$ be any supercomposite of $t$ and $u$; clearly $v$ uniformly witnesses $\gamma \preceq \epsilon$ at $A$ for all $(\gamma, \epsilon) \in T$.

Next, we must equip $D^{C}$ with a suitable evaluation morphism.

Definition 4.8 Let eval : $\mathrm{D}^{\mathrm{C}} \times \mathrm{C} \longrightarrow \mathrm{D}$ be the simulation given as follows.

- The action on types is given by $\operatorname{eval}(F \times A)=U_{F}(A)$.

- For each $F$ and $A$, we define eval $_{F \times A} \subseteq(F \times A) \times U_{F}(A)$ by

$$
\operatorname{eval}_{F \times A}((\gamma, a), b) \Leftrightarrow \gamma_{A}(a, b)
$$

(This is a total relation since each $\gamma_{A}$ is total.)

Proposition 4.9 eval $: \mathrm{D}^{\mathrm{C}} \times \mathrm{C} \longrightarrow \mathrm{D}$ is indeed a simulation.

Proof: We wish to show that every relation $R \times r$ in $\left(\mathrm{D}^{\mathrm{C}} \times \mathrm{C}\right)[F \times A, G \times B]=$ $\mathrm{D}^{\mathrm{C}}[F, G] \otimes \mathrm{C}[A, B]$ is tracked by some $u \in \mathrm{D}\left[U_{F}(A), U_{G}(B)\right]$ with respect to eval. Let $s \in$ $\mathrm{D}\left[U_{F}(A), U_{F}(B)\right]$ track $r$ uniformly with respect to all $\gamma \in F$, and let $t \in \mathrm{D}\left[U_{F}(B), U_{G}(B)\right]$ witness $\gamma \preceq \delta$ at $B$ uniformly for all $(\gamma, \delta) \in R$. Now let $u$ be a supercomposite of $s$ and $t$.

To see that $u$ tracks $R \times r$ with respect to eval, suppose that $((\gamma, a),(\delta, b)) \in(R \times r)$ and $\operatorname{eval}\left((\gamma, a), a^{\prime}\right)$ - that is to say, $(\gamma, \delta) \in R,(a, b) \in r$ and $\gamma_{A}\left(a, a^{\prime}\right)$. We require $b^{\prime} \in \delta B$ such that $u\left(a^{\prime}, b^{\prime}\right)$ and $\operatorname{eval}\left((\delta, b), b^{\prime}\right)$ - that is, $\delta_{B}\left(b, b^{\prime}\right)$. Since $s$ tracks $r$ with respect to $\gamma$, we may find $b^{\prime \prime}$ such that $s\left(a, b^{\prime \prime}\right)$ and $\gamma_{B}\left(b, b^{\prime \prime}\right)$. And since $t$ witnesses $\gamma \preceq \delta$ at $B$, we may find $t\left(b^{\prime \prime}, b^{\prime}\right)$ and $\delta_{B}\left(b, b^{\prime}\right)$. This gives $u\left(a^{\prime}, b^{\prime}\right)$ as required.

It remains to verify that $\left(\mathrm{D}^{\mathrm{C}}\right.$, eval $)$ is a near-exponential for $(\mathrm{C}, \mathrm{D})$. From now on, let $\mathrm{E}$ be some fixed $\mathrm{C}$-structure and $\alpha: \mathrm{E} \times \mathrm{C} \longrightarrow \mathrm{D}$ some fixed simulation.

For any $E \in|\mathrm{E}|$ and any $e \in E$, let us write $\alpha_{e}$ for the simulation $\mathrm{C} \longrightarrow \mathrm{D}$ defined by

$$
\alpha_{e} A=\alpha(E \times A) \quad \alpha_{e A}(c, d) \Leftrightarrow \alpha_{E \times A}((e, c), d)
$$

To see that $\alpha_{e}$ is a simulation, suppose $r \in \mathrm{C}[A, B]$. Let $i$ be a superidentity in $\mathrm{E}[E, E]$; then any $t \in \mathrm{D}[\alpha(E \times A), \alpha(E \times B)]$ that tracks $i \times r$ with respect to $\alpha$ also tracks $r$ with respect to $\alpha_{e}$. Since such a $t$ may be chosen independently of $e$, the family $\left\{\alpha_{e} \mid e \in E\right\}$ is uniformly tracked. This justifies the following definition: 
Definition 4.10 Let $\bar{\alpha}: \mathrm{E} \longrightarrow \mathrm{D}^{\mathrm{C}}$ be defined as follows (we call $\bar{\alpha}$ the transpose of $\alpha$ ).

- For each $E \in|\mathrm{E}|$, let $\bar{\alpha} E=\left\{\alpha_{e} \mid e \in E\right\} \in\left|\mathrm{D}^{\mathrm{C}}\right|$.

- For $E \in|\mathrm{E}|$ and $e \in E$, take $\bar{\alpha}_{E}(e, \gamma)$ iff $\gamma=\alpha_{e}$.

Proposition $4.11 \bar{\alpha}$ is a single-valued simulation, and eval $\circ\left(\bar{\alpha} \times \mathrm{id}_{\mathrm{C}}\right)=\alpha$ on the nose.

Proof: That $\bar{\alpha}$ is single-valued is immediate from the definition. To see that $\bar{\alpha}$ is a simulation, suppose $r \in \mathrm{E}[E, F]$, and let $R=\left\{\left(\alpha_{e}, \alpha_{f}\right) \mid(e, f) \in r\right\}$. We first show that $R$ is a uniform transformation. Given any $A \in|\mathrm{C}|$, let $i \in \mathrm{C}[A, A]$ be a superidentity, and take $r^{\prime} \in \mathrm{D}[\alpha(E \times A), \alpha(F \times A)]$ tracking $r \times i$ with respect to $\alpha$. We claim that for any $(e, f) \in r, r^{\prime}$ tracks $\alpha_{e} \preceq \alpha_{f}$ at $A$. For suppose $\alpha_{e}(c, d)$; then $\alpha((e, c), d)$, so there exists $g$ with $r(d, g)$ and $\alpha((f, c), g)$, whence $\alpha_{f}(c, g)$. Thus $R \in \mathrm{D}^{\mathrm{C}}[\bar{\alpha} E, \bar{\alpha} F]$.

Next, we claim that $R$ tracks $r$ with respect to $\bar{\alpha}$. For suppose $r(e, f)$ and $\bar{\alpha}(e, \gamma)$; then $\gamma=\alpha_{e}$, so taking $\delta=\alpha_{f}$ we have $\bar{\alpha}(f, \delta)$ and $R(\gamma, \delta)$.

To see that eval $\circ\left(\bar{\alpha} \times \mathrm{id}_{\mathrm{C}}\right)=\alpha$, note that if $E \in|\mathrm{E}|, C \in|\mathrm{C}|$ and $D=\alpha(E \times C)$ then

$$
\begin{aligned}
\left(\operatorname{eval} \circ\left(\bar{\alpha} \times \operatorname{id}_{C}\right)\right)_{E \times C}((e, c), d) & \Leftrightarrow \quad e v a l_{\bar{\alpha} E \times C}\left(\left(\alpha_{e}, c\right), d\right) \\
& \Leftrightarrow \alpha_{e C}(c, d) \\
& \Leftrightarrow \alpha_{E \times C}((e, c), d)
\end{aligned}
$$

This already establishes that $\mathcal{C S T} \mathcal{R U C \mathcal { T }}$ is at least weakly cartesian closed. To conclude our proof that $\mathcal{C S T} \mathcal{R U C T}$ has near-exponentials, we need the following:

Proposition 4.12 Suppose $\bar{\alpha}^{\prime}: \mathrm{E} \longrightarrow \mathrm{D}^{\mathrm{C}}$ is any single-valued simulation such that eval $\circ$ $\left(\bar{\alpha}^{\prime} \times \operatorname{id}_{\mathrm{C}}\right)=\alpha$. Then $\bar{\alpha} \preceq \bar{\alpha}^{\prime} \preceq \bar{\alpha}$.

Proof: Suppose $\bar{\alpha}^{\prime}$ is as above. For any $E \in|\mathrm{E}|$ and $e \in E$, write $\alpha_{e}^{\prime}$ for the unique simulation $\mathrm{C} \longrightarrow \mathrm{D}$ such that $\bar{\alpha}_{E}^{\prime}\left(e, \alpha_{e}^{\prime}\right)$. Then as in the proof of Proposition 4.11, we have $\alpha_{e C}^{\prime}(c, d)$ iff $\alpha_{e C}(c, d)$. So $\alpha_{e}^{\prime}=\alpha_{e}$, though $\bar{\alpha} E$ and $\bar{\alpha}^{\prime} E$ may be different. However, we have shown that $\bar{\alpha}^{\prime}\left(e, \alpha_{e}\right)$ for each $e$, so we know that $\bar{\alpha} E=\left\{\alpha_{e} \mid e \in E\right\} \subseteq \bar{\alpha}^{\prime} E$. So we may consider $\bar{\alpha} E \times \bar{\alpha} E$ as a subset of $\bar{\alpha} E \times \bar{\alpha}^{\prime} E$, or of $\bar{\alpha}^{\prime} E \times \bar{\alpha} E$. In either case, $\bar{\alpha} E \times \bar{\alpha} E$ is a uniform transformation, being witnessed by superidentities everywhere, and so is an element of both $\mathrm{D}^{\mathrm{C}}\left[\bar{\alpha} E, \bar{\alpha}^{\prime} E\right]$ and $\mathrm{D}^{\mathrm{C}}\left[\bar{\alpha}^{\prime} E, \bar{\alpha} E\right]$. The first of these witnesses $\bar{\alpha} \preceq \bar{\alpha}^{\prime}$ at $E$; the second $\bar{\alpha}^{\prime} \preceq \bar{\alpha}$ at $E$. Since $E \in|E|$ was arbitrary, we have $\bar{\alpha} \preceq \bar{\alpha}^{\prime} \preceq \bar{\alpha}$.

This completes the proof of Theorem 4.5. However, it is worth noting that a little more may be said about the morphism $\bar{\alpha}$ :

Proposition 4.13 Suppose $\bar{\alpha}^{\prime}: \mathrm{E} \longrightarrow \mathrm{D}^{\mathrm{C}}$ is any simulation (not necessarily single-valued) such that eval $\circ\left(\bar{\alpha}^{\prime} \times \operatorname{id}_{\mathrm{C}}\right)=\alpha$. Then $\bar{\alpha}^{\prime} \preceq \bar{\alpha}$.

Proof: Suppose $\bar{\alpha}^{\prime}$ is as above. Given $E \in|\mathrm{E}|$ and $e \in \mathrm{E}$, let $\epsilon$ be any simulation $\mathrm{C} \longrightarrow \mathrm{D}$ such that $\bar{\alpha}_{E}^{\prime}(e, \epsilon)$. Then we have the sequence of implications

$$
\begin{aligned}
\epsilon_{C}(c, d) & \Rightarrow \operatorname{eval}_{\bar{\alpha}^{\prime} E \times C}((\epsilon, c), d) \\
& \Rightarrow\left(\operatorname{eval} \circ\left(\bar{\alpha}^{\prime} \times \mathrm{id}_{C}\right)\right)_{E \times C}((e, c), d) \\
& \Rightarrow \alpha_{E \times C}((e, c), d) \\
& \Rightarrow \alpha_{e C}(c, d)
\end{aligned}
$$


So at any $C \in|C|, \epsilon \preceq \alpha_{e}$ is witnessed at $C$ by a superidentity on $\alpha(E \times C)$, independently of $e$. So let $R \subseteq \alpha^{\prime} E \times \alpha E$ be the relation $\left\{\left(\epsilon, \alpha_{e}\right) \mid e \in E,(e, \epsilon) \in \bar{\alpha}_{E}^{\prime}\right\}$; then $R$ is a uniform transformation and witnesses $\bar{\alpha}^{\prime} \preceq \bar{\alpha}$ at $E$. Since $E$ is arbitrary, we have $\bar{\alpha}^{\prime} \preceq \bar{\alpha}$.

We remark in passing that even for relatively simple $C$ and $D$, the structure of $D^{C}$ may be wildly intractable. For instance, if $K_{1}$ denotes Kleene's first model (the PCA of natural numbers with Kleene application) then $K_{1}^{K_{1}}$ would appear to be at least as complicated as the lattice of Turing degrees, probably much worse. A possibility for a radically cut-down version of our near-exponentials will be briefly mentioned in Section 6.2.

Finally, it is natural to ask whether our near-exponential construction can be adapted to yield genuine exponentials in some suitable subcategory. Indeed (as suggested by the anonymous referee) one can achieve something of this kind by working in the 2-category of C-structures and single-valued simulations. In this case, one should of course define $\left|D^{C}\right|$ to consist only of uniformly tracked and inhabited families of single-valued simulations; once this is done, the evaluation morphism is indeed single-valued, as is the transpose $\bar{\alpha}$ of a single-valued morphism $\alpha$. However, this still only makes $D^{C}$ a 'pseudo-exponential', since the uniqueness property of $\bar{\alpha}$ holds only up to $\preceq \succeq$. (Of course, one can then get a category with genuine exponentials by quotienting modulo $\preceq \succeq$ if so desired.) Moreover, the possible conceptual significance of this 'single-valued exponential' is at present no clearer to us than that of the full one.

\section{Some subcategories of $\mathcal{C S} \mathcal{T} \mathcal{R U C T}$}

There are many natural subcategories of $\mathcal{C S T} \mathcal{R U C T}$ that one might consider. In [12, Chapter 2] we showed how certain specific properties of PCAs and of applicative morphisms were reflected by categorical properties of the corresponding categories and functors; here we see how a similar though somewhat richer story may be told for C-structures and simulations.

Sections 5.1-5.3 explore some simple properties of C-structures and of simulations that do not involve any additional structure on the class of datatypes. In Section 5.1 we investigate the alternative treatment of non-determinism alluded to in Remark 3.6(ii) above this leads us to the 2-category of tight C-structures and tight simulations between them. In Section 5.2 we consider the important subclass of deterministic C-structures (those in which all operations are partial functions) - here the tight/non-tight distinction vanishes completely. In Section 5.3, we revisit two properties of simulations that played an important role in [12, Chapter 2], namely discreteness and projectivity (here called univalence). As a culmination of this section, we obtain a useful property of equivalences between C-structures: these always consist of discrete, univalent simulations.

In the remaining two subsections, we discuss C-structures whose class of datatypes carries some additional structure. In Section 5.4 we briefly consider C-structures equipped with product types; this is a necessary preliminary to Section 5.5, where we develop the theory of higher-order C-structures. It is here that we make good an earlier promise and show how the theory of TPCAs may be recovered as a special case of our more general theory: roughly speaking, deterministic higher-order C-structures correspond closely to relative TPCAs. 


\section{$5.1 \quad$ Tightness}

We begin by exploring the alternative approach to non-determinism mentioned earlier. The basic idea here is that if a relation $r^{\prime}$ is meant to 'simulate' some relation $r$, it is reasonable to require that $r^{\prime}$ admits only as much non-determinism as $r$ does; a simulation with this property is called tight. As we shall see, a satisfactory analogue of our entire theory may be obtained by consistently 'tightening' all our definitions, and whilst this involves a little more technical detail, in some respects this alternative theory enjoys more pleasant properties than the original. (Our use of the term 'tight' is similar in spirit to, though technically different from, its use in [5].)

Definition 5.1 (i) A C-structure C is called tight if

- for any $A \in|\mathrm{C}|$, the identity relation $\mathrm{id}_{A}$ is present in $\mathrm{C}[A, A]$;

- for any $r \in \mathrm{C}[A, B]$ and $s \in \mathrm{C}[B, C]$, there exists $t \in \mathrm{C}[A, C]$ such that

$$
a \in \operatorname{dom}(r ; s) \Rightarrow(t(a, c) \Leftrightarrow \exists b \cdot r(a, b) \wedge s(b, c))
$$

(ii) A simulation $\gamma: \mathrm{C} \longrightarrow \mathrm{D}$ is tight if every $r \in \mathrm{C}[A, B]$ is tightly tracked by some $r^{\prime} \in \mathrm{D}[\gamma A, \gamma B]$ : that is, $r^{\prime}$ tracks $r$ in the usual sense, and

$$
r(a, b) \wedge \gamma\left(a, a^{\prime}\right) \wedge r^{\prime}\left(a^{\prime}, b^{\prime}\right) \Rightarrow \gamma\left(b, b^{\prime}\right)
$$

(iii) Given $\gamma, \delta: \mathrm{C} \longrightarrow \mathrm{D}$, we say $\gamma$ is tightly transformable to $\delta$, and write $\gamma \preceq_{t} \delta$, if for each $A \in|\mathrm{C}|$ there exists $t \in \mathrm{D}[\gamma A, \delta A]$ such that

$$
\gamma_{A}\left(a, a^{\prime}\right) \Rightarrow\left(\exists a^{\prime \prime} . t\left(a^{\prime}, a^{\prime \prime}\right)\right) \wedge\left(\forall a^{\prime \prime} . t\left(a^{\prime}, a^{\prime \prime}\right) \Rightarrow \delta_{A}\left(a, a^{\prime \prime}\right)\right)
$$

A tight C-structure still need not be closed under relational composition, since in part (i) of the above definition the domain of $t$ may be larger than that of $r ; s$. Whilst in principle one could consider tight simulations between non-tight $\mathrm{C}$-structures, there would appear to be little merit in doing so. It is routine to check that tight C-structures, tight simulations and tight transformations between them constitute a preorder-enriched category, which we denote by $\mathcal{C S T} \mathcal{R U C T}{ }_{t}$. We say $\mathrm{C}, \mathrm{D}$ are tightly equivalent, and write $\mathrm{C} \simeq_{t} \mathrm{D}$, if they are equivalent in $\mathcal{C S T} \mathcal{R} \mathcal{H C} \mathcal{T}_{t}$.

We may also apply the concept of tightness to morphisms of assemblies.

Definition 5.2 A morphism $f: X \rightarrow Y$ in $\mathbf{A s m}(\mathrm{C})$ is called tight if it is tightly tracked by some $r \in \mathrm{C}\left[A_{X}, A_{Y}\right]$ : that is, $r$ tracks $f$ in the usual sense, and

$$
a \Vdash_{X} x \wedge r(a, b) \Rightarrow b \Vdash_{Y} f(x)
$$

If $C$ is tight, it is easy to check that assemblies and tight morphisms constitute a wide subcategory of $\mathbf{A s m}(\mathrm{C})$, which we denote by $\mathbf{A s m}_{t}(\mathrm{C})$. The relationship with tight simulations is given by the following: 
Proposition 5.3 (i) Suppose C, D are tight C-structures. A simulation $\gamma: \mathrm{C} \longrightarrow \mathrm{D}$ is tight iff $\gamma_{*}=\operatorname{Asm}(\gamma)$ maps tight morphisms to tight morphisms.

(ii) Suppose $\gamma, \delta: \mathrm{C} \longrightarrow \mathrm{D}$ are tight simulations between tight $C$-structures, and $\gamma \preceq \delta$. Then $\gamma \preceq_{t} \delta$ iff all components of $\operatorname{Asm}(\gamma \preceq \delta)$ are tight morphisms.

Proof: (i) Suppose $\gamma$ is tight. If $r$ tightly tracks $f: X \rightarrow Y$ in $\operatorname{Asm}(\mathrm{C})$, and $r^{\prime}$ tightly tracks $r$ with respect to $\gamma$, it is easy to check that $r^{\prime}$ tightly tracks $\gamma_{*}(f)$ in $\operatorname{Asm}(D)$. Conversely, suppose $\gamma_{*}$ preserves tight morphisms, and suppose $r \in \mathrm{C}[A, B]$. Define assemblies $X, Y$ as follows:

$|X|=|Y|=\operatorname{dom} r, \quad A_{X}=A, \quad A_{Y}=B, \quad a^{\prime} \Vdash_{X} a \Leftrightarrow a^{\prime}=a, \quad b \Vdash_{Y} a \Leftrightarrow r(a, b)$

Let $f: X \rightarrow Y$ be the identity function on dom $r$; clearly this is tightly tracked by $r$ itself. So $\gamma_{*}(f)$ is tightly tracked by some $r^{\prime}$, and it is easy to see that this $r^{\prime}$ tightly tracks $r$ with respect to $\gamma$.

(ii) Let $\xi=\operatorname{Asm}(\gamma \preceq \delta)$. First, suppose $\gamma \preceq_{t} \delta$, and consider the morphism $\xi_{X}$ for an arbitrary assembly $X \in \mathbf{A s m}(\mathrm{C})$. Take $t \in \mathrm{D}\left[\gamma A_{X}, \delta A_{X}\right]$ that tightly witnesses $\gamma \preceq \delta$ at $A_{X}$; then $t$ tightly tracks $\xi_{X}$. Conversely, suppose every $\xi_{X}$ is tight, and consider an arbitrary $A \in|\mathrm{C}|$. Let $Z_{A}$ be the object of realizers given by $\left|Z_{A}\right|=A, A_{Z_{A}}=A, \Vdash_{Z_{A}}=\mathrm{id}_{A}$, and suppose $r$ tightly tracks $\xi_{Z_{A}}$; then $r$ tightly witnesses $\gamma \preceq \delta$ at $A$.

In fact, by considering $\mathbf{A s m}_{t}(\mathrm{C})$ as a category in its own right, we may obtain a 'tight' analogue of our theory which makes no reference to $\operatorname{Asm}(C)$. If $C$ is tight, then clearly all the subobject, quotient and copy projection morphisms appearing in the proof of Proposition 3.11 are tight, and it is easy to see that the relevant universal properties still hold within $\mathbf{A s m}_{t}(\mathrm{C})$. So $\left(\mathbf{A s m}_{t}(\mathrm{C}), \Gamma_{\mathrm{C}}\right)$ is a quasi-regular category. Moreover, the above proposition shows that if $\gamma$ is a tight simulation between tight C-structures, then $\operatorname{Asm}(\gamma)$ restricts to a functor $\mathbf{A s m}_{t}(\gamma): \mathbf{A s m}_{t}(\mathbf{C}) \rightarrow \mathbf{A s m}_{t}(\mathbf{D})$, and that if $\gamma \preceq{ }_{t} \delta$ then $\mathbf{A s m}(\gamma \preceq \delta)$ constitutes a natural transformation between such functors. We thus have a 2 -functor

$$
\mathbf{A s m}_{t}: \mathcal{C} \mathcal{S T} \mathcal{R U C T} \mathcal{T}_{t} \rightarrow \Gamma \mathcal{Q R E \mathcal { G }}
$$

Furthermore, the analogue of Theorem 3.20 holds:

Theorem 5.4 The 2-functor $\mathbf{A s m}_{t}$ is locally an equivalence. Hence $\mathrm{C} \simeq_{t} \mathrm{D}$ iff $\mathbf{A s m}_{t}(\mathrm{C})$, $\mathbf{A s m}_{t}(\mathrm{D})$ are equivalent in $\Gamma \mathcal{Q R \mathcal { E }}$.

Proof: The proof of Theorem 3.20 goes through in the tight setting with minor adjustments.

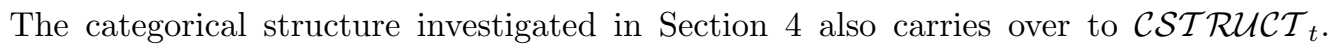
Firstly, $\mathcal{C S} \mathcal{S} \mathcal{R C} \mathcal{T}_{t}$ clearly inherits the finite products and sums described in Propositions 4.1 and 4.2 , so that the inclusion $\mathcal{C S T R U C T}{ }_{t} \rightarrow \mathcal{C S T} \mathcal{R U C T}$ preserves this structure. Secondly, $\mathcal{C S T} \mathcal{R} \mathcal{U C} \mathcal{T}_{t}$ possesses near-exponentials analogous to, but different from, those of $\mathcal{C S T R U C T}$, so that these are not preserved by the inclusion. Specifically, to construct $\mathrm{D}^{\mathrm{C}}$ within $\mathcal{C} \mathcal{S} \mathcal{T} \mathcal{R} \mathcal{C} \mathcal{T}_{t}$, one should take $\left|\mathrm{D}^{\mathrm{C}}\right|$ to be the set of all inhabited and uniformly tightly tracked families of simulations $\mathrm{C} \longrightarrow \mathrm{D}$, and $\mathrm{D}^{\mathrm{C}}[F, G]$ to be the set of uniform tight transformations $R \subseteq F \times G$. The proof of Theorem 4.5 then goes through mutatis mutandis. 
A few minor gaps in this story should be noted. There does not seem to be a simple categorical characterization of the tight assembly morphisms among those of $\operatorname{Asm}(\mathrm{C})$, nor of those functors that preserve tight morphisms. Likewise, the property that $\mathrm{C}$ is tight does not seem to be mirrored by any particular categorical property of $\mathbf{A s m}(\mathrm{C})$; indeed, we conjecture that a tight $\mathrm{C}$-structure may be equivalent in $\mathcal{C S T} \mathcal{R U C T}$ to a non-tight one.

\subsection{Deterministic C-structures}

A class of C-structures it is very natural to consider consists of those that model deterministic flavours of computation. We here show that this is a mathematically well-behaved class from the point of view of our theory.

Definition 5.5 A C-structure $\mathrm{C}$ is deterministic if for any $r \in \mathrm{C}[A, B]$ we have

$$
r(a, b) \wedge r\left(a, b^{\prime}\right) \Rightarrow b=b^{\prime}
$$

Thus, deterministic C-structures are those in which the 'relations' are partial functions. We write $r \cdot a$ for the unique $b$ such that $r(a, b)$ if one exists. For such structures, the tight/non-tight distinction evaporates completely. For example, if $D$ is deterministic then not only is $\mathrm{D}$ tight, but every simulation $\mathrm{C} \longrightarrow \mathrm{D}$ is tight, as is every transformation $\gamma \preceq \delta$ where $\gamma, \delta: \mathrm{C} \longrightarrow \mathrm{D}$. Furthermore:

Proposition 5.6 A C-structure $\mathrm{D}$ is deterministic iff $\mathbf{A s m}(\mathrm{D})=\mathbf{A s m}_{t}(\mathrm{D})$.

Proof: If $\mathbf{D}$ is deterministic and $r$ tracks $f: X \rightarrow Y$ in $\mathbf{A s m}(\mathrm{D})$, then for any $a \Vdash_{X} x$ there is exactly one $b$ with $r(a, b)$, so $r$ tracks $f$ tightly and $f \in \mathbf{A s m}_{t}(\mathrm{D})$. Conversely, if D is not deterministic, take $r \in \mathrm{D}[A, B]$ and $a \in A, b, b^{\prime} \in B$ with $r(a, b), r\left(a, b^{\prime}\right)$ and $b \neq b^{\prime}$, and consider the assemblies $X, Y$ defined by

$|X|=|Y|=\left\{b, b^{\prime}\right\}, \quad A_{X}=A, \quad A_{Y}=B, \quad a^{\prime} \Vdash_{X} x \Leftrightarrow a^{\prime}=a, \quad b^{\prime \prime} \Vdash_{Y} y \Leftrightarrow b^{\prime \prime}=y$

Then the identity map $X \rightarrow Y$ is tracked by $r$, but cannot be tightly tracked by any $r^{\prime} \in \mathrm{D}[A, B]$.

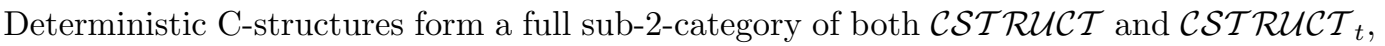
which we denote by $\mathcal{C S T R U C T}$. Moreover, the class of deterministic $\mathrm{C}$-structures is closed under equivalences in $\mathcal{C S T} \mathcal{R U C T} \mathcal{T}_{t}$ :

Proposition 5.7 If $\mathrm{D}$ is deterministic and $\mathrm{C} \simeq \mathrm{D}$ in $\mathcal{C S T} \mathcal{R U C T} \mathcal{T}_{t}$, then $\mathrm{C}$ is deterministic.

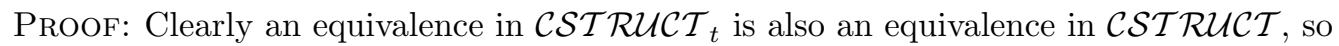
by the 2-functoriality of $\mathbf{A s m}_{t}$ and $\mathbf{A s m}$, if $\mathrm{C} \simeq \mathrm{D}$ in $\mathcal{C S T R \mathcal { C } T} \mathcal{T}_{t}$ then both $\mathbf{A s m}_{t}(\mathrm{C}) \simeq$ $\operatorname{Asm}_{t}(\mathrm{D})$ and $\operatorname{Asm}(\mathrm{C}) \simeq \operatorname{Asm}(\mathrm{D})$. Moreover, the equivalences are easily seen to commute with the inclusions $\mathbf{A s m}_{t}(-) \rightarrow \mathbf{A s m}(-)$. But if $\mathbf{D}$ is deterministic then $\mathbf{A s m}_{t}(\mathrm{D})$ is the whole of $\mathbf{A s m}(\mathrm{D})$, whence $\mathbf{A s m}_{t}(\mathrm{C})=\mathbf{A} \mathbf{s m}(\mathrm{C})$ and $\mathrm{C}$ is deterministic by Proposition 5.6. 
This says in effect that 'determinism' is indeed an invariant property of a computability notion with respect to tight equivalences. We do not know whether this is the case for equivalences in $\mathcal{C S T} \mathcal{R U C T}$.

Deterministic C-structures are generally pleasant to work with since they allow many of the basic definitions to be significantly simplified. For instance, treating relations as partial functions, the notion ' $r$ ' tracks $r$ with respect to $\gamma$ ' may be expressed as

$$
\gamma_{A}\left(a, a^{\prime}\right) \wedge r(a) \downarrow \Rightarrow \gamma_{B}\left(r \cdot a, r^{\prime} \cdot a^{\prime}\right)
$$

and ' $t$ witnesses $\gamma \preceq \delta$ at $A$ ' may be expressed as

$$
\gamma_{A}\left(a, a^{\prime}\right) \Rightarrow \gamma_{A}\left(a, t \cdot a^{\prime}\right)
$$

The definition of morphism in $\mathbf{A s m ( C )}$ may similarly be simplified.

Clearly the class of deterministic C-structures is closed under finite products and sums. Unfortunately they are not closed under the near-exponentials of Definition 4.6 (or their tight counterparts); however, it turns out that $\mathcal{C S} \mathcal{S} \mathcal{R U C} \mathcal{T}_{d}$ is endowed with its own 'nearexponentials' in a somewhat weaker sense. Let us here say a simulation $E \times C \longrightarrow D$ is left-injective if for any $E \in|\mathrm{E}|$, the simulations $\alpha_{e}$ for $e \in E$ are all distinct.

Theorem 5.8 If C, D are deterministic C-structures, there exist a deterministic $C$-structure $\mathrm{F}$ and a left-injective simulation eval $: \mathrm{F} \times \mathrm{C} \longrightarrow \mathrm{D}$ with the following property: for any left-injective $\alpha: \mathrm{E} \times \mathrm{C} \longrightarrow \mathrm{D}$ there is a single-valued simulation $\bar{\alpha}: \mathrm{E} \longrightarrow \mathrm{F}$ such that eval $\circ\left(\bar{\alpha} \times \mathrm{id}_{\mathrm{C}}\right)=\alpha$, and moreover $\bar{\alpha}$ is unique up to $\preceq \succeq$ among simulations with this property.

Proof Sketch: Take $|\mathrm{F}|$ to be the set of all inhabited, uniformly tracked families of simulations as before, and for $F, G \in|\mathrm{F}|$, define $\mathrm{F}[F, G]$ to be the set of single-valued uniform transformations $R \subseteq F \times G$. The rest of the argument then proceeds essentially as before; the significance of left-injectivity is that in the proof of Proposition 4.11, we need to know that if $r \in \mathrm{F}[E, F]$ is single-valued then so is $R=\left\{\left(\alpha_{e}, \alpha_{f}\right) \mid(e, f) \in r\right\}$.

Note that even this weaker universal property is still sufficient to characterize the Cstructure F up to equivalence, so that we may speak of the near-exponentiation of deterministic C-structures as a well-defined operation on deterministic notions of computability.

\subsection{Discrete and univalent morphisms}

Among the properties of simulations considered in [12] in the PCA setting were two properties known as discreteness and projectivity. We here consider the analogues of both these properties, changing the name of the latter to univalence, for reasons to be explained. Each of these properties of simulations is naturally correlated with a property of assemblies.

Definition 5.9 (i) A simulation $\gamma: \mathrm{C} \longrightarrow \mathrm{D}$ is called discrete if $\gamma_{A}(a, b)$ and $\gamma_{A}\left(a^{\prime}, b\right)$ imply $a=a^{\prime}$.

(ii) An assembly $X$ over $C$ is discrete if $a \Vdash_{X} x$ and $a \Vdash_{X} x^{\prime}$ imply $x=x^{\prime}$. (Such assemblies are also known as modest sets.) 
The following is straightforward:

Proposition 5.10 A simulation $\gamma$ is discrete iff $\mathbf{A s m}(\gamma)$ preserves discrete objects.

When $\mathrm{C}$ is tight, the discrete objects may be characterized categorically within $\mathbf{A s m}_{t}(\mathrm{C})$ (whence the functors $\mathbf{A s m}_{t}(\gamma)$ arising from discrete simulations may also be characterized categorically). By a singleton assembly we mean any assembly $U$ such that $\Gamma(U)$ is a singleton set.

Proposition 5.11 Suppose $\mathrm{C}$ is tight. An assembly $X$ over $\mathrm{C}$ is discrete iff for every singleton object $U$ and set $S$, every morphism $U \propto S \rightarrow X$ in $\mathbf{A s m}_{t}(\mathrm{C})$ is constant.

Proof: The forwards implication is straightforward. For the converse, if $X$ is not discrete, take $x \neq x^{\prime}$ and $a$ such that $a \Vdash_{X} x$ and $a \Vdash_{X} x^{\prime}$. Let $U$ be the singleton assembly with just the realizer $a$, let $S$ be a two-element set, and let $f: U \propto S \rightarrow X$ map the two elements of $|U \propto S|$ to $x$ and $x^{\prime}$ respectively. Since C is tight, $f$ is tightly tracked by the appropriate identity relation, and so is a non-constant morphism of $\mathbf{A s m}_{t}(\mathrm{C})$.

There does not appear to be a correspondingly simple characterization of the discrete objects within $\operatorname{Asm}(\mathrm{C})$. It is also worth noting that the tight setting preserves a familiar feature from the classical theory of realizability: namely, that a morphism into a discrete assembly is uniquely determined by any tracker for it.

Two further facts about discrete simulations in the tight setting is worth recording. The proofs are straightforward.

Proposition 5.12 (i) If $\gamma: \mathrm{C} \longrightarrow \mathrm{D}$ and $\delta: \mathrm{D} \longrightarrow \mathrm{C}$ are tight simulations with $\delta \circ \gamma \preceq \mathrm{id}_{\mathrm{C}}$, then $\gamma$ is discrete.

(ii) If $\gamma: \mathrm{C} \longrightarrow \mathrm{D}$ is discrete and $\mathrm{D}$ is deterministic, then $\mathrm{C}$ is deterministic.

Combining the two halves of this yields a useful strengthening of Proposition 5.7.

Next, we consider a property of simulations we shall call univalence. Although the name is perhaps not ideal, the intuition is that these are simulations that are 'essentially singlevalued'. This notion comes in a non-tight and a tight flavour. The notion of a single-valued simulation was defined in Definition 4.3; similarly, we call an assembly $X$ single-valued if for each $x \in|X|$ there is a unique $a$ with $a \Vdash_{X} x$.

Definition 5.13 (i) A simulation $\gamma: \mathrm{C} \longrightarrow \mathrm{D}$ is univalent [resp. tightly univalent] if for some single-valued simulation $\gamma^{\prime}$ we have $\gamma \preceq \gamma^{\prime}$ [resp. $\gamma \preceq_{t} \succeq_{t} \gamma^{\prime}$ ].

(ii) An assembly $X$ over $C$ is univalent [resp. tightly univalent] if for some single-valued assembly $X^{\prime}$ we have $X \cong X^{\prime}$ in $\mathbf{A} \mathbf{s m}(\mathrm{C})$ [resp. in $\mathbf{A s m}_{t}(\mathrm{C})$ ].

Proposition $\mathbf{5 . 1 4}$ (i) $\gamma: \mathrm{C} \longrightarrow \mathrm{D}$ is univalent iff $\mathbf{A s m}(\gamma)$ preserves univalent assemblies.

(ii) $\gamma$ is tightly univalent iff $\mathbf{A s m}_{t}(\gamma)$ preserves tightly univalent assemblies.

Proof: (i) The forwards implication is easy. For the reverse, suppose $\gamma_{*}=\mathbf{A} \mathbf{s m}(\gamma)$ preserves univalent assemblies. For each $A \in|\mathrm{C}|$, let $Z_{A} \in \operatorname{Asm}(\mathrm{C})$ be the corresponding object of realizers, and choose $Y_{A} \in \operatorname{Asm}(\mathrm{D})$ single-valued such that $\left|Y_{A}\right|=A$ and $Y_{A} \cong$ 
$\gamma_{*}\left(Z_{A}\right)$ via the identity on underlying sets. Define $\gamma^{\prime}: \mathrm{C} \longrightarrow \mathrm{D}$ by $\gamma_{A}^{\prime}(a, b) \Leftrightarrow b \Vdash_{Y_{A}} a$. We need to show that $\gamma^{\prime}$ is a simulation; it is then clear that $\gamma^{\prime}$ is single-valued and $\gamma \preceq \succeq \gamma^{\prime}$.

Given $r \in \mathrm{C}[A, B]$, construct the following diagram in $\mathbf{A s m}(\mathrm{C})$ as in the proof of Proposition 3.18:

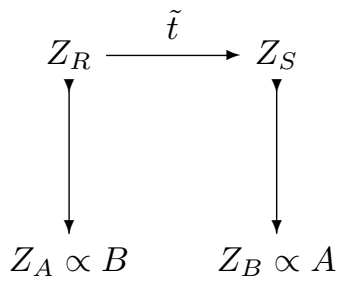

Apply $\gamma_{*}$ to obtain a diagram $\Delta$ in $\mathbf{A s m}(\mathrm{D})$. Now construct a diagram $\Delta^{\prime}$ in $\mathbf{A s m}(\mathrm{D})$ isomorphic to $\Delta$ as follows: replace $\gamma_{*}\left(Z_{A}\right), \gamma_{*}\left(Z_{B}\right)$ by $Y_{A}, Y_{B}$ respectively, then replace the copies and subobjects in turn by the corresponding canonical ones in $\operatorname{Asm}(\mathrm{D})$. Let $f$ be the morphism in $\Delta^{\prime}$ corresponding to $\gamma_{*}(\tilde{t})$ in $\Delta$, and take $r^{\prime}$ tracking $f$. It is easy to see that $r^{\prime}$ tracks $r$ with respect to $\gamma^{\prime}$.

The proof of (ii) is similar.

In contrast to the situation for discrete assemblies, it is the non-tight setting that seems more friendly to univalent assemblies. This is illustrated by the following categorical characterization of univalent objects within $\mathbf{A s m}(\mathrm{C})$ (the proof involves the Axiom of Choice):

Proposition 5.15 An assembly $X$ over $C$ is univalent iff (in $\mathbf{A s m}(\mathrm{C})$ ) for every morphism $f: X \rightarrow Z$ and every quotient morphism $h: Y \rightarrow X$ there exists $\bar{f}: X \rightarrow Y$ such that $h \circ \bar{f}=f$.

Proof: First suppose $X$ is univalent; we may as well assume it is single-valued. Suppose $f$ and $h$ are as above with $f$ tracked by $r$. We define $\bar{f}$ as follows: For each $x \in|X|$, choose some $a \in A_{X}$ and $b \in A_{Z}$ such that $a \Vdash_{X} x, b \Vdash_{Z} f(x)$ and $r(a, b)$; then choose $y \in|Y|$ such that $h(y)=f(x)$ and $b \Vdash_{Y} y$, and set $\bar{f}(x)=y$. Clearly $\bar{f}$ is tracked by $r$ and $h \circ \bar{f}=f$.

For the converse, suppose $X$ has the given quotient property, and consider the assembly $Y$ where $|Y|$ is the relation $\Vdash_{X}$ considered as a set of pairs $(a, x)$, and $a^{\prime} \Vdash_{Y}(a, x)$ iff $a^{\prime}=a$. Let $h: Y \rightarrow X$ be the quotient map given by second projection (this is tracked by a superidentity), and let $f: X \rightarrow X$ be the identity morphism. Take $\bar{f}: X \rightarrow Y$ such that $h \circ \bar{f}=f$. Consider the image of $\bar{f}$ as a subset of $|Y|$, and lift this to a subobject $s: X^{\prime} \longmapsto Y$; then $\bar{f}$ factors through $s$ and we have an isomorphism $X \cong X^{\prime}$ with $X^{\prime}$ single-valued.

Remark 5.16 In the PCA setting, the quotient maps are precisely the regular epis, so the quotient property featuring in the above proposition is precisely the property of being a (regular) projective. For this reason, univalent simulations and assemblies were referred to as projective in [12]. However, we hesitate to adopt this terminology in our present setting, not only because not all quotients need be regular epis in general, but also because there does not appear to be an analogue of the above proposition for $\mathbf{A s m}_{t}(\mathrm{C})$.

The following useful fact generalizes Theorem 2.5.3(ii) of [12]: 
Theorem 5.17 Suppose $\mathrm{D}$ is deterministic. If $\gamma: \mathrm{C} \longrightarrow \mathrm{D}$ and $\delta: \mathrm{D} \longrightarrow \mathrm{C}$ are simulations with $\delta \circ \gamma \preceq \operatorname{id}_{\mathrm{C}}$ and $\operatorname{id}_{\mathrm{D}} \preceq \gamma \circ \delta$, then $\delta$ is univalent.

Proof: For each $B \in|\mathrm{D}|$, take $u_{B} \in \mathrm{D}[B, \gamma \delta B]$ tracking $\operatorname{id}_{\mathrm{D}} \preceq \gamma \circ \delta$ at $B$. Since $\mathrm{D}$ is deterministic, for each $b \in B$ there is a unique $d_{b}$ with $u_{B}\left(b, d_{b}\right)$, and it satisfies $(\gamma \delta)_{B}\left(b, d_{b}\right)$. Moreover, there is a unique $c_{b} \in \delta_{B}$ with $\delta_{B}\left(b, c_{b}\right)$ and $\gamma_{\delta B}\left(c_{b}, d_{b}\right)$, since $\gamma$ is discrete by Proposition 5.12. Define $\delta^{\prime}: \mathrm{D} \longrightarrow \mathrm{C}$ by $\delta_{B}^{\prime}(b, c) \Leftrightarrow c=c_{b}$. We shall show that $\delta, \delta^{\prime}$ are 'intertransformable' as relations, and hence that $\delta^{\prime}$ is a simulation.

Clearly $\delta_{B}^{\prime}(b, c)$ implies $\delta_{B}(b, c)$. Conversely, if $\delta_{B}(b, c)$, take $u_{B}^{\prime} \in \mathrm{C}[\delta B, \delta \gamma \delta B]$ tracking $u_{B}$ with respect to $\delta$; then for some $c^{\prime} \in \delta \gamma \delta B$ we have $u_{B}^{\prime}\left(c, c^{\prime}\right)$ and $\delta_{\gamma \delta B}\left(d_{b}, c^{\prime}\right)$, whence $(\delta \gamma)_{\delta B}\left(c_{b}, c^{\prime}\right)$. Now take $t_{B}$ tracking $\delta \circ \gamma \preceq \operatorname{id}_{c}$ at $\delta_{B}$; then $t_{B}\left(c^{\prime}, c_{b}\right)$. So taking $v_{B} \in$ $\mathrm{C}\left[\delta B, \delta^{\prime} B\right]$ a supercomposite of $u_{B}^{\prime}$ and $t_{B}$, we have that $v_{B}\left(c, c_{b}\right)$. It is now easy to see that $\delta^{\prime}$ is a simulation: for any $r \in \mathrm{D}[A, B]$, take $r^{\prime} \in \mathrm{C}[\delta A, \delta B]$ tracking $r$ with respect to $\delta$, and take $r^{\prime \prime} \in \mathrm{C}\left[\delta^{\prime} A, \delta^{\prime} B\right]$ a supercomposite of $r^{\prime}$ and $v_{B}$ (noting that $\delta^{\prime} A=\delta A$ ); then clearly $r^{\prime \prime}$ tracks $r$ with respect to $\delta^{\prime}$. Moreover, $\delta^{\prime} \preceq \delta$ is tracked at $B$ by a superidentity, and $\delta \preceq \delta^{\prime}$ is tracked at $B$ by $v_{B}$.

Corollary 5.18 If $\mathrm{C} \simeq \mathrm{D}$ in $\mathcal{C} \mathcal{S} \mathcal{T} \mathcal{R U C} \mathcal{T}_{d}$, then both halves of the equivalence are discrete and univalent; hence there is an equivalence $\mathrm{C} \simeq \mathrm{D}$ consisting of single-valued simulations.

In the light of Remark 3.6(ii), this shows that for deterministic C-structures, our notion of equivalence agrees precisely with the lax simulation equivalence of [5] (taking sets and partial functions as the base category). As shown in [12, Chapter 3], knowing that equivalences, when they exist, can be assumed to be single-valued is often helpful for proving the inequivalence of two models.

Note also that if C, D are strict deterministic C-structures, the above argument can be adapted to show that an equivalence consisting of strict simulations can be replaced by one consisting of strict single-valued simulations; the resulting notion thus agrees with the strict simulation equivalence of [5].

\subsection{C-structures with products}

We now turn to consider C-structures in which the class of datatypes carries some additional structure.

We regard it as a matter of some pride that our theory so far has not required our C-structures to be endowed with products of any kind. Nevertheless, most naturally arising C-structures do have products of some kind, so it is natural to consider various possible notions of product structure. This is also a necessary prerequisite for the notion of a higher order C-structure, which we investigate in the next subsection.

We opt here for a simple notion of product types with a relatively 'strict' flavour.

Definition 5.19 Suppose C is a C-structure.

(i) We say $\mathrm{C}$ is strict monoidal if

- $|\mathrm{C}|$ contains the singleton set $I=\{*\}$ and is closed under set-theoretic binary products;

- for any $r \in \mathrm{C}[A, B]$ and $s \in \mathrm{C}[C, D]$, the relation ' $r \times s$ ' is present in $\mathrm{C}[A \times C, B \times D]$; 
- the canonical bijections $(A \times B) \times C \cong A \times(B \times C), I \times A \cong A$ and $A \times I \cong A$ are all present as relations in $\mathrm{C}$ in both directions.

(ii) We say $\mathrm{C}$ has strict cartesian if, in addition to the above, the canonical projection and diagonal maps $A \times B \rightarrow A, A \times B \rightarrow B, A \rightarrow A \times A$ are present as relations in $\mathrm{C}$.

Other more relaxed notions of monoidal or cartesian C-structure may be devised, but it is typically the case that any such C-structure will be equivalent to a strict one (we refrain from going into details here). For example, all TPCAs (including untyped PCAs) are equivalent to strict cartesian C-structures, although they may not be such structures themselves.

Clearly, if $\mathrm{C}$ is strict monoidal [resp. strict cartesian] then $\mathbf{A s m}(\mathrm{C})$ is a monoidal [resp. cartesian] category (and similarly for $\mathbf{A s m}_{t}(\mathrm{C})$ in the case that $\mathrm{C}$ is tight).

The relevant structure-respecting simulations for strict monoidal C-structures are the following:

Definition 5.20 Suppose C, D are strict monoidal. A simulation $\gamma: \mathrm{C} \longrightarrow \mathrm{D}$ is monoidal if

- for any $A, B \in|\mathrm{C}|$ there is a relation $t \in \mathrm{D}[\gamma A \times \gamma B, \gamma(A \times B)]$ such that

$$
\gamma_{A}\left(a, a^{\prime}\right) \wedge \gamma_{B}\left(b, b^{\prime}\right) \Rightarrow \exists c^{\prime} . t\left(\left(a^{\prime}, b^{\prime}\right), c^{\prime}\right) \wedge \gamma_{A \times B}\left((a, b), c^{\prime}\right)
$$

- there exists $u \in \mathrm{D}[I, \gamma I]$ such that $u(*, a)$ for some $a \Vdash_{I} *$.

Recall that if $(\mathcal{C}, \otimes, I)$ and $\left(\mathcal{D}, \otimes^{\prime}, I^{\prime}\right)$ are monoidal categories, a lax monoidal functor between them is a functor $F: \mathcal{C} \rightarrow \mathcal{D}$ together with a family of morphisms $\phi_{A, B}: F(A) \otimes^{\prime}$ $F(B) \rightarrow F(A \otimes B)$, natural in $A$ and $B$, and a morphism $\psi: I^{\prime} \rightarrow F(I)$, such that certain coherence conditions involving the associativity and unit morphisms are satisfied. We record the following easy fact without proof:

Proposition 5.21 Suppose C, D are strict monoidal. A simulation $\gamma: \mathrm{C} \longrightarrow \mathrm{D}$ is monoidal iff $\operatorname{Asm}(\gamma)$ is lax monoidal.

Note also that if the products in C, D are cartesian, then a lax monoidal functor $\mathbf{A s m}(\gamma)$ is automatically cartesian (i.e. preserves finite products).

We may write $\mathcal{C S} \mathcal{S} \mathcal{R} \mathcal{U C} \mathcal{T}_{m}$ for the preorder-enriched category of strict monoidal Cstructures and monoidal simulations. We say $\mathrm{C}, \mathrm{D}$ are monoidally equivalent $\left(\mathrm{C} \simeq_{m} \mathrm{D}\right)$ if

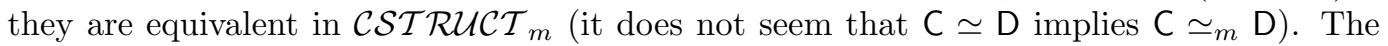
2-category $\mathcal{C S T R U C T}{ }_{m}$ has finite products as in $\mathcal{S} \mathcal{S T} \mathcal{R U C T}$, and it is routine to verify that a monoidal analogue of the construction of near-exponentials goes through. Note, however, that our simple construction of finite sums is not available in $\mathcal{C S \mathcal { T R U C T }}{ }_{m}$, since $\mathrm{C}+\mathrm{D}$ will not typically contain products $A \times B$ where $A \in|\mathrm{C}|, B \in|\mathrm{D}|$.

Finally, we remark that analogous results hold in the 'tight' setting. If $C$ and $D$ are tight and strict monoidal, we may say a tight simulation $\gamma: \mathrm{C} \longrightarrow \mathrm{D}$ is tightly monoidal if it satisfies the obvious tightened version of Definition 5.20. We then have that $\gamma$ is tightly monoidal iff $\mathbf{A s m}_{t}(\gamma)$ is lax monoidal; such a functor is automatically cartesian if C and D are. The corresponding 2-category $\mathcal{C S T R U C \mathcal { T }}{ }_{m t}$ enjoys properties similar to $\mathcal{C S} \mathcal{T} \mathcal{R U C T}{ }_{m}$. 


\subsection{Higher order C-structures}

With the machinery of products now in place, we may now identify a class of C-structures that model higher order notions of computation, and thus retrieve the theory of TPCAs as a special case of our theory. The key idea here is that a C-structure is higher order if its computable operations can themselves be represented as values of an appropriate type.

Definition 5.22 $A$ higher order C-structure is a strict monoidal $C$-structure $\mathrm{C}$ equipped with the following: for each $A, B \in|\mathrm{C}|$, a datatype $(A \Rightarrow B) \in|\mathrm{C}|$ and an operation $\operatorname{app}_{A B} \in \mathrm{C}[(A \Rightarrow B) \times A, B]$ such that

$$
\begin{aligned}
& \text { for any } r \in \mathrm{C}[C \times A, B] \text { there exists a total relation } \bar{r} \in \mathrm{C}[C,(A \Rightarrow B)] \text { with } \\
& r \subseteq\left(\bar{r} \times \operatorname{id}_{A}\right) ; \operatorname{app}_{A B} .
\end{aligned}
$$

It is crucial here that $\bar{r}$ is not required to be unique; we may therefore say $a p p_{A B}$ enjoys a 'weak universal property'.

It is not hard to show that if $\mathrm{C}$ is a C-structure [resp. cartesian C-structure] then $\mathbf{A s m}(\mathrm{C})$ is monoidal closed [resp. cartesian closed]. Thus, as is often the case in realizability, the Asm construction turns a weak universal structure into a strong one.

Any TPCA $C$ may be turned into a deterministic, higher order cartesian C-structure $\operatorname{CStr}(C)$ as follows. First, it is easy to construct some $C^{\prime}$ equivalent to $C$ (within $\mathcal{T} \mathcal{P C} \mathcal{A}$ ) in which product types are genuine set-theoretic products, and in which a unit type exists. Such a $C^{\prime}$ may then be viewed as a C-structure $\operatorname{CStr}(C)$ as in Example 3.3(i). Clearly $\operatorname{CStr}(C)$ is deterministic and cartesian, and for any types $A, B$, the element

$$
\lambda^{*} p .(\text { fst } p)(\text { snd } p) \in(((A \Rightarrow B) \times A) \Rightarrow B)
$$

induces a computable operation $\operatorname{app}_{A B}:(A \Rightarrow B) \times A \rightarrow B$ with the required weak universal property. (We assume familiarity here with the $\lambda^{*}$ notation for combinators - see e.g. [12, Chapter 1].)

In one important respect, however, deterministic higher order cartesian C-structures are significantly more general than TPCAs. In C-structures of the form $\mathbf{C S t r}(C)$, every element $a$ of any $A \in|\mathrm{C}|$ is picked out by some operation in $\mathrm{C}[I, A]$ (We may call such elements computable, since the intuition is that mappings in $\mathrm{C}[I, A]$ are 'computable operations'.) In general, however, this need not be the case - rather, the elements picked out by such operations will constitute a sub-C-structure of $\mathrm{C}$ in an evident sense. This amounts to the observation that deterministic higher order cartesian C-structures naturally embrace the concept of relative realizability (see e.g. [3]). Informally, the idea behind relative realizability is to consider (for instance) a TPCA $A$ equipped with a sub-TPCA $A^{\sharp}$ of elements we regard as 'computable'. Under certain strictness assumptions, ${ }^{5}$ it can be shown that deterministic higher order cartesian C-structures correspond exactly to TPCAs equipped with such a choice of sub-TPCA.

In any case, this convergence between TPCAs and certain C-structures suggests how the notion of applicative morphism for TPCAs (as in Section 2.2) might be generalized to arbitrary higher order C-structures:

\footnotetext{
${ }^{5}$ Specifically, strict TPCAs endowed with such a sub-TPCA correspond precisely to deterministic higher order C-structures in which the operation $\bar{r}$ satisfies $r=\left(\bar{r} \times \operatorname{id}_{A}\right) ; a p p_{A B}$.
} 
Definition 5.23 Suppose C, D are higher order C-structures. An applicative morphism $\gamma: \mathrm{C} \rightarrow \mathrm{D}$ consists of a mapping $A \mapsto \gamma A:|\mathrm{C}| \rightarrow|\mathrm{D}|$ and a family of total relations $\gamma_{A} \subseteq A \times \gamma A$ (for $\left.A \in|C|\right)$ satisfying the following conditions:

1. For every computable element $a \in A$ (where $A \in|C|$ ) there is some computable $a^{\prime} \in \gamma A$ such that $\gamma\left(a, a^{\prime}\right)$.

2. 'Application in $\mathrm{C}$ is tracked in $\mathrm{D}$ ': that is, for any $A, B \in|\mathrm{C}|$, we may choose app ${ }_{A B} \in$ $\mathrm{C}[(A \Rightarrow B) \times A, B]$ as in Definition 5.22 such that for some app ${ }_{A B}^{\prime} \in \mathrm{D}[\gamma(A \Rightarrow$ $B) \times \gamma A, \gamma B]$ we have

$$
\gamma_{(A \Rightarrow B)}\left(f, f^{\prime}\right) \wedge \gamma_{A}\left(a, a^{\prime}\right) \wedge a p p_{A B}((f, a), b) \Rightarrow \exists b^{\prime} \cdot \gamma_{B}\left(b, b^{\prime}\right) \wedge a p p_{A B}^{\prime}\left(\left(f^{\prime}, a^{\prime}\right), b^{\prime}\right)
$$

If $C, D$ are TPCAs, it is easy to see that applicative morphisms $\operatorname{CStr}(C) \rightarrow \operatorname{CStr}(D)$ in the above sense correspond exactly to applicative morphisms $C \longrightarrow D$ in the sense of Section 2.2. Indeed, if $r \in D_{\gamma(A \Rightarrow B) \Rightarrow \gamma A \Rightarrow \gamma B}$ tracks $\cdot A B$ in the TPCA sense, then $\lambda^{*} p . r(f s t p)($ snd $p)$ induces a suitable operation $a p p_{A B}^{\prime} \in \operatorname{CStr}(D)[(A \Rightarrow B) \times A, B]$; conversely, any such operation $a p p_{A B}^{\prime}$ is induced by some $r^{\prime} \in D_{\gamma(A \Rightarrow B) \times \gamma A \Rightarrow \gamma B}$, and then $\lambda^{*} f a . r^{\prime}$ (pair $\left.f a\right)$ tracks $\cdot A B$ in the TPCA sense. So Definition 5.23 is a natural and direct generalization of the TPCA notion of applicative morphism.

The connection with simulations is now given by the following:

Proposition 5.24 If C, D are higher order C-structures, then the applicative morphisms from $\mathrm{C}$ to $\mathrm{D}$ are exactly the monoidal simulations $\mathrm{C} \longrightarrow \mathrm{D}$.

Proof: First suppose $\gamma$ is a monoidal simulation. To see that condition 1 of Definition 5.23 is satisfied, note that if $a \in A$ is picked out by $r \in \mathrm{C}[I, A]$ and $r$ is tracked by $r^{\prime} \in \mathrm{D}[\gamma I, \gamma A]$, then by supercomposing $r$ with the operation $u \in \mathrm{D}[I, \gamma I]$ from Definition 5.20 yields an operation in $\mathrm{D}[I, \gamma A]$ that picks out a $\gamma$-realizer for $a$. For condition 2, given any $A, B \in|C|$ we have an operation $t \in \mathrm{D}[\gamma(A \Rightarrow B) \times \gamma A, \gamma((A \Rightarrow B) \times A)]$ as in Definition 5.20, and an operation $u \in \mathrm{D}[\gamma((A \Rightarrow B) \times A), \gamma B]$ tracking the operation $a p p_{A B}$ in C. Supercomposing these gives an operation $a p p_{A B}^{\prime} \in \mathrm{D}[\gamma(A \Rightarrow B) \times \gamma A, \gamma B]$ with the required properties.

Conversely, suppose $\gamma$ is an applicative morphism. Given any $r \in \mathrm{C}[A, B]$, supercompose with the bijection in $\mathrm{C}[I \times A, A]$ to obtain $r^{\prime} \in \mathrm{C}[I \times A, B]$, then choose $\bar{r} \in \mathrm{C}[I,(A \Rightarrow B)]$ as in Definition 5.22. Let $f \in(A \Rightarrow B)$ be any computable element picked out by $\bar{r}$; then by condition 1 of Definition 5.23 , we may find $f^{\prime} \in \gamma(A \Rightarrow B)$ picked out by some $t \in \mathrm{D}[I, \gamma(A \Rightarrow B)]$ such that $\gamma_{(A \Rightarrow B)}\left(f, f^{\prime}\right)$. By supercomposing the canonical operation in $\mathrm{D}[A, I \times A]$ with $t \times i \in \mathrm{D}[I \times A,(A \Rightarrow B) \times A]$ and then with $a p p_{A B}^{\prime}$, we obtain an operation in $\mathrm{D}[A, B]$ that tracks $r$.

The basic idea here - that if the application operations are tracked then so are all operations - is one that also plays an important role in [4].

Under the above correspondence, it is easy to see that the preorder $\preceq$ on monoidal simulations agrees precisely with the one on applicative morphisms as in Section 2.2. Thus, the CStr construction outlined above extends to a 2-functor CStr : $\mathcal{T P C A} \rightarrow$ $\mathcal{C S T R U C T}{ }_{m}$ which is locally an equivalence. (Hence two TPCAs $A, B$ are equivalent iff 
$\operatorname{CStr}(A), \operatorname{CStr}(B)$ are equivalent as monoidal C-structures.) Moreover, as noted earlier, the Asm construction on TPCAs agrees with that on C-structures modulo this inclusion.

The observation that the notion of applicative morphism is in essence a 'first order' one highlights a significant philosophical point. One might have supposed that two models of higher order computation could be equivalent as 'flattened' first order models but differ nontrivially on account of the different higher order structures they carried. Proposition 5.24 shows that this is not the case, at least within our present framework: equivalence of higher order models is simply their equivalence qua first order models.

Note, however, that the class of higher order C-structures appears to have poor closure properties by comparison with that of all C-structures. Not only are higher order C-structures not closed under finite sums, but they do not appear to admit an analogue of our near-exponential construction. Curiously, then, it is the shift from higher order to first order structures that allows 'higher order' phenomena to appear at the framework level.

Finally, we remark briefly on the 'tight' counterpart of the above results. The above definition of higher order C-structure is still appropriate in the tight setting, but there is an obvious tight analogue of Definition 5.23 in the case where C, D are tight higher-order C-structures. The analogue of Proposition 5.24 then goes through: the tight applicative morphisms from $\mathrm{C}$ to $\mathrm{D}$ are exactly the tightly monoidal simulations as discussed at the end of Section 5.4. Of course, the ordinary and the tight versions of these results are equally good as generalizations of the theory of TPCAs, since the latter fall within the realm of deterministic C-structures.

\section{Conclusions and further work}

\subsection{Summary of achievements}

In this paper, we have presented a broad framework for the study of models of computation and simulations between them, and shown that the 'category of assemblies' construction and associated correspondence theorem generalize smoothly to this setting. We have also seen that our 2-category of models possesses some non-trivial mathematical structure (most notably a slightly relaxed form of exponentiation), as well as a number of well-behaved subcategories corresponding to various properties of computational interest. Whilst our investigation of this structure has been far from exhaustive, we believe that our results sufficiently demonstrate the richness and fertility of our proposed framework.

We have also made a tentative start on showing how a wide range of existing computation models not previously studied in a realizability context might fit naturally within our framework. In particular, in Section 3 we have seen how a very general class of 'syntactic models' can be regarded as C-structures, and have presented one concrete and substantial example of a simulation, embodying Milner's translation from lazy $\lambda$-calculus to $\pi$-calculus. However, a much broader and deeper investigation would be required to clarify the precise extent of applicability of our framework.

Along the way, we have compared our framework with other possible candidates for a general framework for computability models and simulations, such as those proposed by Cockett and Hofstra $[4,5]$. Some of the differences are rather technical in nature, and we have not arrived at any definitive arguments in favour of one approach over another: indeed, 
as regards finding the 'right' level of generality for these ideas it may be that we are still far from the end of the story. Nevertheless, the broad picture that emerges is that our category $\mathcal{C S T} \mathcal{R U C T}$ offers a more general setting than other current contenders (which can be naturally viewed as subcategories of it) — with the notable exception of its 'ordered' counterpart (cf. [22, 7]) which would appear to be a straightforward structural enrichment of our framework. It remains open whether this ordered version admits any additional examples of computational interest.

Finally, we have shown how the higher order framework represented by the theory of TPCAs can be naturally subsumed within a first order framework. Indeed, it now appears that higher order structure plays a less vital role than we previously supposed, since the same notion of (monoidal) simulation, and hence of equivalence, is equally appropriate in both the first order and higher order settings. Combined with the observation that the class of higher order C-structures does not enjoy particularly good closure properties, we are led to the view that higher order models should really be regarded as nothing more than first order models that happen to satisfy a certain completeness condition - a moral of some significance for our general programme of mapping out computability notions.

\subsection{Further work}

It is likely that there is further categorical structure to be uncovered within $\mathcal{C S T} \mathcal{R U C T}$. Certainly there are other subcategories of interest that we have not discussed here. For example, it is natural to look at C-structures that contain a pair of elements playing the role of the booleans. The relevant boolean-respecting simulations between such structures correspond to what are called decidable morphisms in [12]. ${ }^{6}$ The subcategory of such simulations would itself appear to have reasonable structure; moreover, it admits a cut-down version of the near-exponential construction which in typical cases yields a much more tractable structure than the one in $\mathcal{C S T} \mathcal{R U C \mathcal { T }}$. (For instance, the near-exponential $K_{1}^{K_{1}}$ in the category of boolean-respecting simulations is the one-element C-structure.) Similarly, one could restrict to C-structures that contain a good representation of the natural numbers, and simulations that respect them (these play an important role in [16]).

Beyond this, the main outstanding task is to populate our abstract framework with some interesting examples (outside the domain of higher order C-structures, which has already received significant attention), and to investigate more fully the extent of its applicability. It seems to us that the realm of process calculi offers a suitable domain in which to begin such an investigation, and our example involving the $\pi$-calculus makes a modest start on this. In the first instance, we would expect that Milner's translation from call-by-value $\lambda$-calculus to $\pi$-calculus, as well as other translations in a similar spirit (see e.g. [19]), should furnish further examples of simulations. However, one should also note that it is at present far from clear whether the construction given in Example 3.4(ii) represents the 'best' way of building a C-structure based on the $\pi$-calculus. Other possible constructions, and the relationships between them, certainly deserve investigation.

One might also look at other process calculi, such as Milner's CCS or Hoare's CSP, and possible translations between them. This would lead naturally to an investigation of the

\footnotetext{
${ }^{6}$ It is remarked in [23] that the name 'decidable' was not very well chosen — a point we are happy to concede. Perhaps 'boolean-respecting' would be more appropriate.
} 
equivalence or otherwise of different models of concurrent computation. In our view, either of the following would represent an interesting advance:

- An example of two known process calculi or models that are not prima facie equivalent but can be shown to yield equivalent C-structures.

- A proof of the inequivalence of two calculi or models that sheds some interesting light on the essential difference in computational power between them.

If the study of process calculi from the point of view of $\mathcal{C S T} \mathcal{R M C T}$ proves a success, one might then broaden the investigation to embrace other paradigms such as quantum computation or membrane computation.

One ulterior motive for this entire project is of course the search for natural 'computability notions' underpinning existing models of computation. For instance, if several superficially different computation models turned out to yield equivalent C-structures, this might be seen as evident that the underlying notion of computability that these represented was somehow a fundamental one. There is thus the hope that our general programme of identifying and classifying natural computability notions (as exemplified in $[15,13,16]$ ) might eventually be extended to a much wider field.

Whether or not this ambitious goal turns out to realizable, we are hopeful that our theory might play some role in bringing together a wide range of current approaches to modelling computation under a single roof, thus enabling us to grasp how they all fit together. In particular, our notion of simulation would seem to offer a promising way of talking about how the views of a computational system at different 'levels of abstraction' are related to one another. Already, the typed PCA framework has proved itself capable of offering such a unifying perspective over a large tract of territory (see [16]); in the long run, we are hopeful that this may be even more true for C-structures. In this way, one can envisage our framework, or something like it, as playing a useful organizational role within theoretical computer science, perhaps somewhat akin to that of category theory itself.

\section{References}

[1] S. Abramsky, The lazy lambda calculus. In D. Turner (ed.), Research Topics in Functional Programming, Addison-Wesley, 65-116 (1989).

[2] S. Abramsky, Process realizability. In F.L. Bauer and R. Steinbrüggen (eds.), Foundations of Secure Computation, IOS Press, 167-180 (2000).

[3] L. Birkedal and J. van Oosten, Relative and modified relative realizability. Annals of Pure and Applied Logic 118, 115-132 (2002).

[4] J.R.B. Cockett and P.J.W. Hofstra, Introduction to Turing categories. Annals of Pure and Applied Logic 156(2-3), 183-209 (2008).

[5] J.R.B. Cockett and P.J.W. Hofstra, Categorical simulations. Journal of Pure and Applied Algebra 214(10), 1835-1853 (2010).

[6] S. Feferman, A language and axioms for explicit mathematics. In Algebra and Logic, Lecture Notes in Mathematics 450, Springer, 87-139 (1975). 
[7] P.J.W. Hofstra, All realizability is relative. Math. Proc. Camb. Phil. Soc. 141, 239-264 (2006).

[8] N. Hoshino, Linear realizability. In Computer Science Logic 200\%, Lecture Notes in Mathematics 4646 of LNCS, Springer, 420-434 (2007).

[9] J.M.E. Hyland, The effective topos. In L.E.J. Brouwer Centenary Symposium, NorthHolland, 165-216 (1982).

[10] J.M.E. Hyland, First steps in synthetic domain theory. In Carboni et al, eds., Category Theory, proceedings, Como 1990, Lecture Notes in Mathematics 1488, Springer, 131156 (1990).

[11] P. Lietz and T. Streicher, Impredicativity entails untypedness. Math. Struct. Comp. Sci. 12, 335-347 (2002).

[12] J.R. Longley, Realizability Toposes and Language Semantics. PhD thesis, University of Edinburgh, 1995.

[13] J.R. Longley, Matching typed and untyped realizability. In L. Birkedal et al (eds.), Proceedings of Workshop on Realizability, Trento. Electronic Notes in Theoretical Computer Science 23(1), Elsevier (1999).

[14] J.R. Longley, Unifying typed and untyped realizability. Unpublished electronic note, available from the author's web page (1999).

[15] J.R. Longley, Notions of computability at higher types I. In R. Cori et al (eds.), Logic Colloquium 2000, Lecture Notes in Logic 19, ASL, 32-142 (2005).

[16] J.R. Longley, On the ubiquity of certain total type structures. Math. Struct. Comp. Sci. 17(5), 841-953 (2007).

[17] G. Longo and E. Moggi, Constructive natural deduction and its ' $\omega$-set' interpretation. Math. Struct. Comp. Sci. 1, 215-254 (1991).

[18] R. Milner, Functions as processes. Math. Struct. Comp. Sci. 2, 119-141 (1992).

[19] D. Sangiorgi, Expressing Mobility in Process Algebras: First-Order and Higher-Order Paradigms. PhD thesis, University of Edinburgh, 1993.

[20] M. Schönfinkel, Über die Bausteine der Mathematischen Logik. Mathematische Annalen 92, 305-316 (1924). Translated in J. van Heijenoort, A Source Book in Mathematical Logic, 1879-1931, Harvard University Press, 344-366 (1967).

[21] P. Taylor, The fixed point property in synthetic domain theory. In Proc. 6th Annual Symposium on Logic in Computer Science, IEEE, 152-160 (1991).

[22] J. van Oosten, Extensional realizability. Annals of Pure and Applied Logic 84, 317-349 (1997).

[23] J. van Oosten, Realizability: An Introduction to its Categorical Side. Studies in Logic and the Foundations of Mathematics 152, Elsevier, 2008. 